\title{
Long- and Short-Term Temporal Variability in Cloud Condensation Nuclei Spectra in the Southern Great
} Plains

Russell J. Perkins, ${ }^{1}$ Peter J. Marinescu,,${ }^{1,2}$ Ezra J. T. Levin, ${ }^{1,3}$ Don R. Collins, ${ }^{4}$ and Sonia M. Kreidenweis ${ }^{1}$

${ }^{1}$ Colorado State University, Fort Collins, CO, 80523, USA

$5 \quad{ }^{2}$ Cooperative Institute for Research in the Atmosphere, Fort Collins, CO, 80523, USA

${ }^{3}$ Handix Scientific, Boulder, CO, 80301, USA

${ }^{4}$ University of California Riverside, Riverside, CA 92521

Correspondence to: Russell J. Perkins (rperkins@colostate.edu)

\section{Abstract.}

When aerosol particles seed formation of liquid water droplets in the atmosphere, they are called cloud condensation nuclei (CCN). Different aerosols will act as $\mathrm{CCN}$ under different degrees of water supersaturation (relative humidity above $100 \%$ ) depending on their size and composition. In this work we build and analyze a best-estimate CCN spectrum product, tabulated at 45 min resolution, generated using high quality data from eight independent instruments at the US Department of Energy Atmospheric Radiation Measurement (ARM) Southern Great Plains site. The data product spans a large 15 supersaturation range, from 0.0001 to $\sim 30 \%$, and time period, 5 years from 2009-2013 and is available on the ARM data archive. We leverage this added statistical power to examine relationships that are unclear in smaller datasets. Probability distributions of many aerosol and CCN metrics are found to exhibit skewed log-normal distribution shapes. Clustering analyses of CCN spectra reveal that the primary drivers of CCN differences are aerosol number size distributions, rather than hygroscopicity or composition, especially at supersaturations above $0.2 \%$, while also allowing for simplified understanding of seasonal and diurnal variations in $\mathrm{CCN}$ behaviour. The predictive ability of using limited hygroscopicity data with accurate number size distributions to estimate CCN spectra is investigated and uncertainties of this approach are estimated. Finally, the dynamics of $\mathrm{CCN}$ spectral clusters and concentrations are examined with cross-correlation and autocorrelation analyses, which assist in determining the time scales of changing $\mathrm{CCN}$ concentrations at different supersaturations and are important for cloud modelling studies.

\section{Introduction}

The interactions between atmospheric aerosol particles and ambient water vapor are key drivers of the formation of haze and clouds. Particles are the nuclei upon which liquid water first condenses to form haze and cloud droplets, thereby affecting visibility, cloud microphysical properties, and precipitation. Water uptake by particles depends upon their size and chemical composition as well as on ambient environmental conditions, and on their rates of change. Particles that are identified as cloud 
lower, conditions believed typical of most clouds that are formed in weak to moderate updrafts. Based on average aerosol characteristics, and typical atmospheric aerosol number size distributions, number concentrations of particles active as $\mathrm{CCN}$ are thus generally assumed to correspond to those particles in the 80-300 nm dry diameter size range. Instruments designed to directly measure number concentrations of activated particles at fixed $S S_{w}$ are also typically limited to $2 \% S S_{w}$ as an upper bound on the measurement range (Uin, 2016).

However, supersaturations and particle sizes outside of these ranges are also of atmospheric interest. In deep convection with intense updrafts, and in regions of very low existing particle or droplet surface area concentrations, $S S_{w}$ of water vapor can build rapidly to high levels as the condensation sink rates are so low relative to the rate of supersaturation generation (Pinsky et al., 2012). Those high $S S_{w}$ may be sufficient to allow particles smaller than 40 nm to serve as cloud condensation nuclei. Thus, despite their relatively short lifetimes in the atmosphere compared with larger accumulation-mode particles, high concentrations of small particles can potentially influence cloud microphysical processes leading to precipitation formation or evaporation. It is now recognized that nucleation of new particles from the gas phase, generating particles on the order of 10 $\mathrm{nm}$ in diameter which subsequently grow, occurs in many regions of the troposphere and is an important control on global atmospheric aerosol number concentrations (Hodshire et al., 2016; Bianchi et al., 2016; Venzac et al., 2008; Pierce et al., 2014; Nieminen et al., 2018).

At the other end of atmospherically-relevant supersaturations, droplet formation may occur at very low $S S_{w}$ where $\mathrm{CCN}$ concentrations are very difficult to probe. For example, the slow cooling rates in radiation fogs allow vapor scavenging to effectively compete with the generation of supersaturation, and thus maximum $S S_{w}$ conditions reached in fogs can be below 0.05\% (Gerber, 1991; Low, 1975; Shen et al., 2018), suggesting that only larger and more hygroscopic particles can participate

50 in fog droplet formation. The cloud physics community has had a longstanding interest in elucidating the microphysical roles of "giant CCN" (GCCN), that is, relatively large particles that activate at very low $S S_{w}, \sim 0.01 \%$ or less. Specifically, GCCN are hypothesized to control the initiation of drizzle and precipitation in shallow clouds (Cohard et al., 1998; Johnson, 1982; Feingold et al., 1999; Cheng et al., 2009; Hudson et al., 2011; Posselt and Lohmann, 2008; Levin and Cotton, 2009; Gantt et al., 2014; Jung et al., 2015).

Their potentially controlling roles in fog, cloud, and precipitation formation has motivated interest in direct measurements of the number concentrations of $\mathrm{CCN}$ active over a range of atmospherically-relevant $S S_{w}$, with modern instrumentation making possible long-term, unattended monitoring. Here, we analyse observations of CCN spectra from the United States Department of Energy's Atmospheric Radiation Measurement's (ARM) Southern Great Plains (SGP) site located in north central Oklahoma for the five-year period from 2009-2013. The CCN measurement instrumentation deployed at this site is

60 typically limited to stable operation over the 0.1 to $1 \% S S_{w}$ range. As described further below, we extend those observations to a broader supersaturation range of interest using ancillary aerosol observations, creating a CCN estimate relevant for clouds 
ranging from fog through to intense deep convective updrafts. These observations are especially important for modelling studies of aerosol impacts on clouds in this region that use $\mathrm{CCN}$ number concentrations as a basis for determining their aerosol initial conditions (Saleeby et al., 2016; Marinescu et al., 2017; Glenn et al., 2020).

\section{Methods}

This work builds upon prior work reported in Marinescu et al. (2019). In that study, for the same 2009-2013 time period studied herein, aerosol data from three instruments deployed at the SGP site were averaged over $\sim 45$-minute intervals and merged into dry aerosol number size distributions, $n\left(D_{p}\right)$, spanning $7 \mathrm{~nm}<$ particle dry diameter, $D_{p}<14 \mu \mathrm{m}$. We build upon that work by combining those merged size distributions with information on aerosol hygroscopicity, $\kappa$ (Petters and

70 Kreidenweis, 2007), taken over the same $\sim 45$-minute intervals. $\kappa$ values were obtained via measurements of diameter growth factors (GF) at $90 \%$ relative humidity measured by a humidified Tandem Differential Mobility Analyzer (H-TDMA; Collins, 2010; Mahish and Collins, 2017). The procedure for integrating these data into a CCN spectrum is described more fully in Appendix A, but is reviewed briefly here.

The critical saturation ratio, $S_{c}$, at which a particle with dry particle diameter $D_{p}$ can be activated into a cloud drop is determined by finding the maximum of the following equation (Petters and Kreidenweis, 2007):

$$
\begin{gathered}
S(D)=\frac{D^{3}-D_{p}^{3}}{D^{3}-D_{p}^{3}(1-\kappa)} \exp \left(\frac{A}{D}\right) \\
A=\frac{4 \sigma_{s / a} M_{w}}{R T \rho_{w}}
\end{gathered}
$$

where $D$ is the droplet diameter, $\rho_{w}$ is the density of water, $M_{w}$ is the molecular weight of water, $\sigma_{s / a}$ is the surface tension of the solution/air interface, $R$ is the universal gas constant, and $T$ is temperature. Saturation ratio, $S$, and water supersaturation percent, $S S_{w}$ are related by:

$$
S S_{w}=(S-1) * 100 \quad \text { Eq. } 3
$$

80 The functional relationship between $D_{p}, \kappa$, and $S_{c}$ can be more readily illustrated by the approximate relationship that is valid for $\kappa>0.2$ (Petters and Kreidenweis, 2007) where $S S_{w, c}$ is the critical water supersaturation percent: 


$$
\frac{S S_{w, c}}{100} \cong\left\{\frac{4 A^{3}}{27 D_{p}^{3} \kappa}\right\}^{1 / 2}
$$

Eq. 4

From Eq. 4 it can be seen that information on the aerosol number size distribution, $n\left(D_{p}\right)$, and the variation of $\kappa$ with dry diameter, can be used to compute the critical $S S_{w}$ corresponding to each selected dry size used in Eq. 4 . It should be noted that the approximation in Eq. 4 is only used for demonstration here, with full methods described in Appendix A. In practice, the size distribution is discretized to obtain total number concentrations in each selected dry diameter bin, 222 logarithmically spaced bins in this work, and a constant $\kappa$ is assumed across each selected bin. To produce the size-dependent $\kappa$ distribution, measurements of aerosol hygroscopic growth were made for 7 different sizes (Figure A2), which were subsequently processed to obtain a single weighted $\kappa$ value for each size. In this study, these $\kappa$ values are then interpolated linearly between measured values, with invariant $\kappa$ beyond the largest and smallest sizes (below $\sim 10 \mathrm{~nm}$ and above $\sim 600 \mathrm{~nm}$ ). The cumulative spectrum of $\mathrm{CCN}$ concentration, $\mathrm{CCN}\left(S S_{w}\right)$, can then be constructed, which defines the total number of particles that can be activated at a particular $S S_{w}$. Figure 1a shows an example of the measured $n\left(D_{p}\right)$ and $\kappa\left(D_{p}\right)$, and the resulting CCN spectrum is shown in Figure 1b.
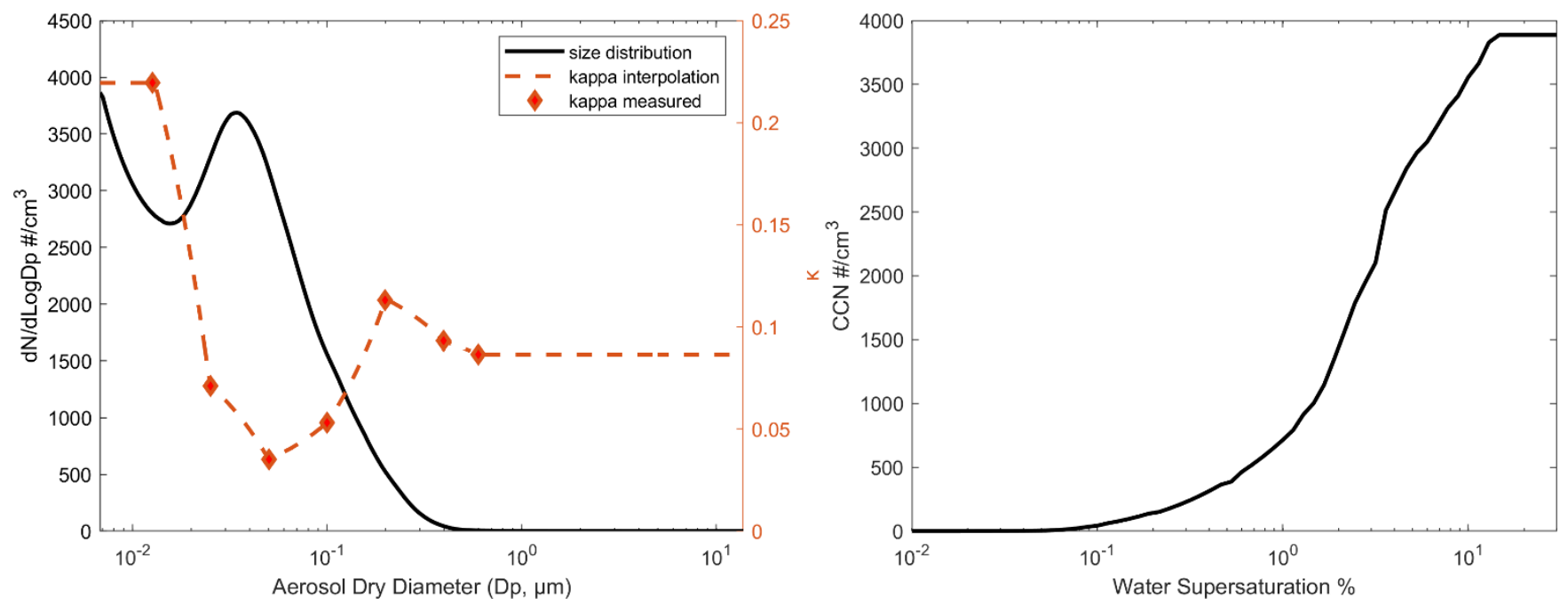

95 Figure 1: Example data products: size (left, blue solid line), $\kappa$ (left, orange dashed line), and CCN (right) distributions. Measured kappa points are shown with markers with linearly interpolated values as dashed lines.

Available data from the measurement suite include direct, concurrent, and collocated observations of cumulative CCN number concentrations at selected supersaturations, which can be used to check the accuracy of the reconstructed spectra. Two additional instruments provide separate, independent, continuous observations that were also used to assess the reconstructed 
spectra: a nephelometer measured total particle scattering coefficients, and an Aerosol Chemical Speciation Monitor (ACSM) measured nonrefractory, speciated submicron mass concentrations. Both of these observations emphasize larger particle sizes (generally > $300 \mathrm{~nm}$ ) and thus served as constraints on the particles contributing strongly at the lowest supersaturations, for which no direct observations exist. At the other end of the size distribution, the smallest particles are expected to require the highest supersaturations for activation, but neither the size distribution nor $\kappa$ are well constrained observationally. Reasonable assumptions are applied to extrapolate the $\mathrm{CCN}$ spectrum beyond $S S_{w}=1 \%$, the approximate upper limit of the $\mathrm{CCN}$ counter. In total, data from six instruments are merged to produce a best-estimate CCN spectrum for each $\sim 45$ minute interval, as described more fully in Appendix A. Resulting size distributions range from 0.0068 to $13.8 \mu \mathrm{m}$ (bin centers), with corresponding CCN spectra generated for $S S_{w}$ from 0.0001 to $\sim 30$ percent $S S_{w}$ (100.0001 to 130 percent RH). The $S S_{w}$ range is chosen to span the entire range of particle activations: the largest and most hygroscopic measured particles activate at

$1100.0001 \% S S_{w}$ while the smallest and least hygroscopic at $\sim 30 \% S S_{w}$. As noted in the Data Availability statement below, the final merged data, with estimates of uncertainty, are available in the DOE ARM archive.

\section{Discussion}

\subsection{Distribution Characteristics}

While we expect the final merged data over the entire range to be useful, all observations are not equally reliable for

115 several reasons. In the size distributions, the lowest size bins are generated using a fitting procedure previously described by Marinescu et al. (2019). This fitting procedure is constrained by instrument data, and produces good agreement with direct observations of the number concentrations of particles in the ultrafine mode (Marinescu et al., 2019), but the shape of the aerosol spectrum at the smallest particle sizes, especially below $12 \mathrm{~nm}$, is more uncertain than at larger particle sizes. $\kappa$ values are assumed to be invariant outside of the measured range (below $\sim 10 \mathrm{~nm}$ and above $\sim 600 \mathrm{~nm}$ ) and are thus more uncertain in those ranges. Additionally, at larger aerosol sizes, growth factor distributions can be bi-modal (Figure A2) which is not captured in this approach, and results in additional uncertainty. Because the CCN spectra are generated by combining size and $\kappa$ distributions, uncertainties in each are inherited in certain regions. The high $S S_{w}$ region of the CCN spectrum is dominated by smaller particles, and uncertainty in this region is increased due to uncertainties in aerosol distribution shape. Beyond a certain $S S_{w}$ that is sufficient to activate all particles regardless of size and composition, a CCN spectrum must level off. In cases where there are particles present in the smallest size bins, this region has increased uncertainty due to the effects of very small particles (below $\sim 8 \mathrm{~nm}$ ) that were not measured. This occurs at $S S_{w}$ greater than $10 \%$. Interestingly, uncertainties in $\kappa$ in this region are largely irrelevant as high $S S_{w}$ is required for activation regardless of hygroscopicity (see discussion in section 3.1.2, Figure 4). The region of lowest $S S_{w}$, less than $0.1 \%$, is the other region subject to additional uncertainties, coming from uncertainties in $\kappa$ and number size distributions. Particles $>13.8 \mu \mathrm{m}$ were not measured, and are not included in our size

130 distributions, and these particles this lowest $S S_{w}$ region. While the abundances of large particles which activate in this low $S S_{w}$ 5 
regime are quite low, they can be important in controlling further activation under some conditions. Ultimately, the regions of increased uncertainty are under $S S_{w}$ conditions where few measurements of CCN concentrations exist, which adds value to these data despite these uncertainties.

\subsubsection{Probability Fitting}

We present statistical descriptions for key parameters related to the aerosol and CCN populations to describe their variability over the study period. Figure 2 shows occurrence probability (y axis) of the number concentration of CCN active at $1 \% S S_{w}$. Similar distribution shapes are observed for all variables examined (total distribution number concentrations, total distribution volume concentrations, number concentrations in 100 and $1000 \mathrm{~nm}$ individual bins, CCN concentrations at $0.01 \%$ and $0.1 \%$ supersaturation; Appendix B).

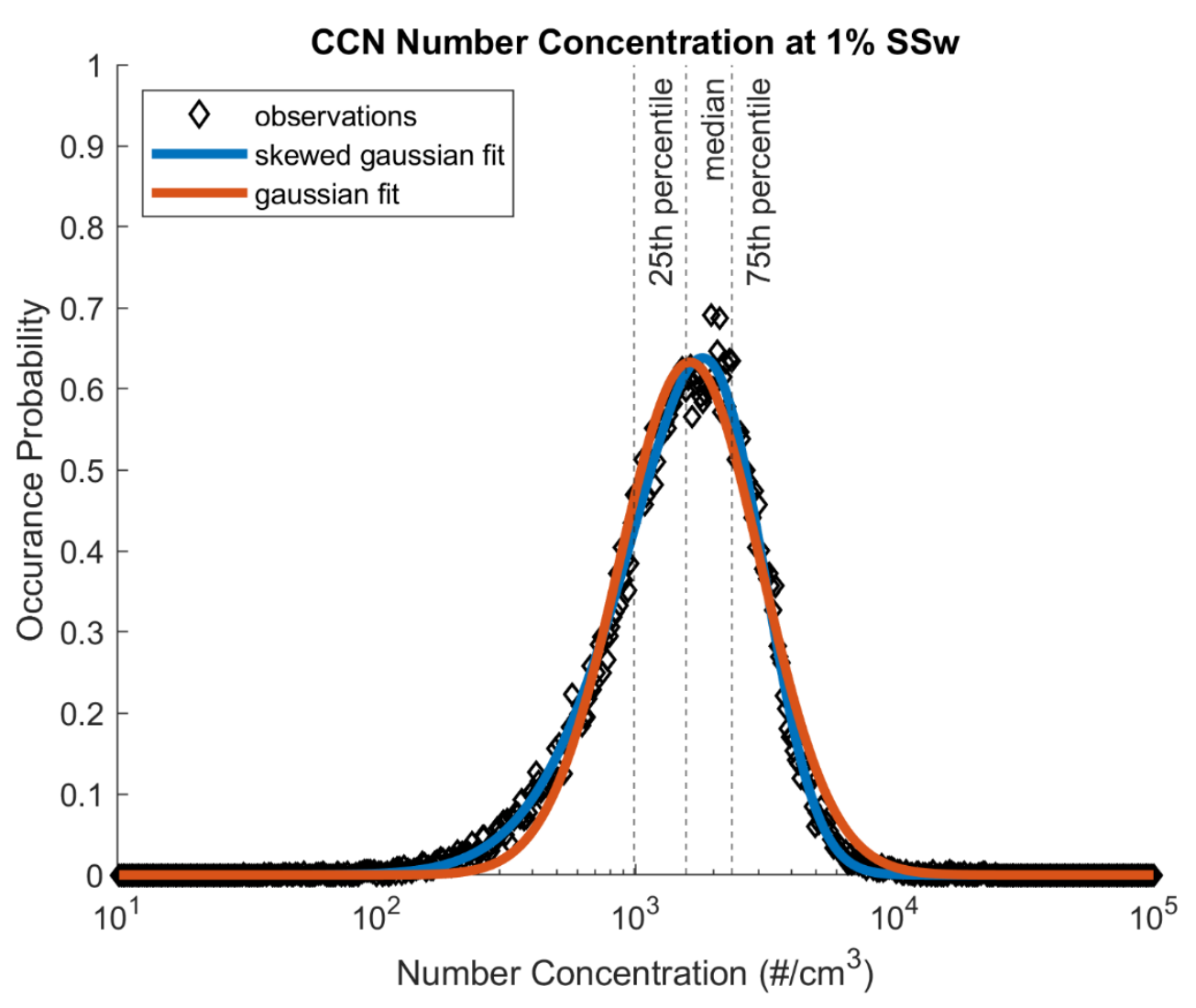

Figure 2: Distribution of CCN number concentrations active at $1 \% S S_{w}$ over the course of the study. The distribution fits a skewed Gaussian model exceptionally well, and an un-skewed Gaussian model with moderate fidelity.

The frequency distributions of observations fit exceptionally well to skewed log-normal distributions (described in Appendix B), with low degrees of skew such that log-normal distributions remain a fair approximation in many cases. Aerosol 6 
145 data are seldom fit in this manner, simply reporting median and percentile bounds instead. On the other hand, log-normal distributions have been noted and used for Aerosol Optical Depth (AOD), either for spatial or temporal variations (Alexandrov et al., 2004, 2016; Anderson et al., 2003; Sayer and Knobelspiesse, 2019). While the methods used for AOD treatments have not been widely adopted for aerosol distributions or $\mathrm{CCN}$ spectra, they could be, and hopefully supplying parameterizations in the same form makes further work that is focused on impacts of variability more accessible. Additionally, better fits for probability distribution functions could be incorporated into microphysical modelling studies or other efforts interested in the likelihood of given aerosol conditions occurring. When using these fit data, it is important to keep in mind that neighbouring size bins are statistically correlated with each other - the probability of finding 100 particles in the $10 \mathrm{~nm}$ size bin is not independent of the probability of finding 100 particles in the $15 \mathrm{~nm}$ size bin. Because of this, the simplest way to calculate combined or correlated quantities (for example, the number concentrations of all particles between 10 and $20 \mathrm{~nm}$ ) is through

155 our archived distributions across time points of interest, rather than utilizing our fit parameters. A similar skewed log-normal distribution could be fit for the combined data if desired. It should also be noted that distribution shapes may not be well conserved across all time or length scales. Variations are most likely to occur at small time (less than 2 hours) or length (less than $0.5 \mathrm{~km}$ ) scales based on the analysis discussed in Section 3.2 and previous works (Alexandrov et al., 2004; Anderson et al., 2003).

\section{3.1.2 Clustering Analysis}

Clustering analysis is used to simplify and seek relationships in the rather large and complicated data set. K-means clustering is performed using a vector-based distance metric. Details of cluster analysis can be found in Appendix C. From the K-means clustering applied to the CCN spectra, three distinct clusters are identified that achieve good separation in both CCN and number size distribution characteristics, as shown in Figure 3. 
ㅁ

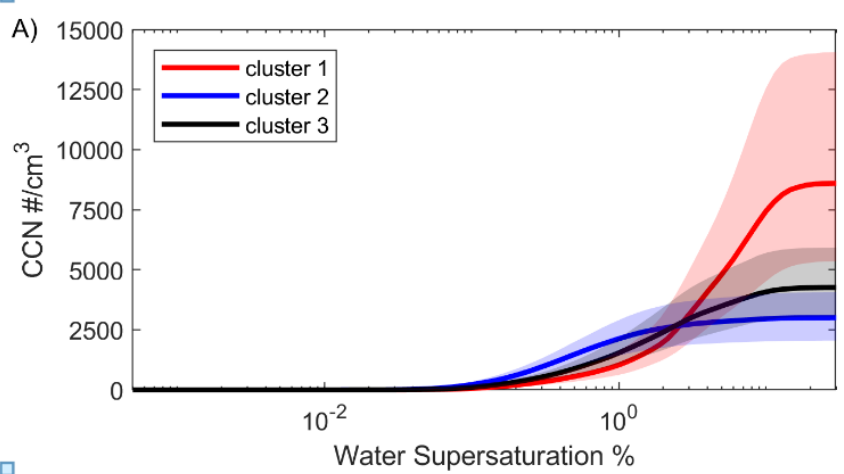

C)

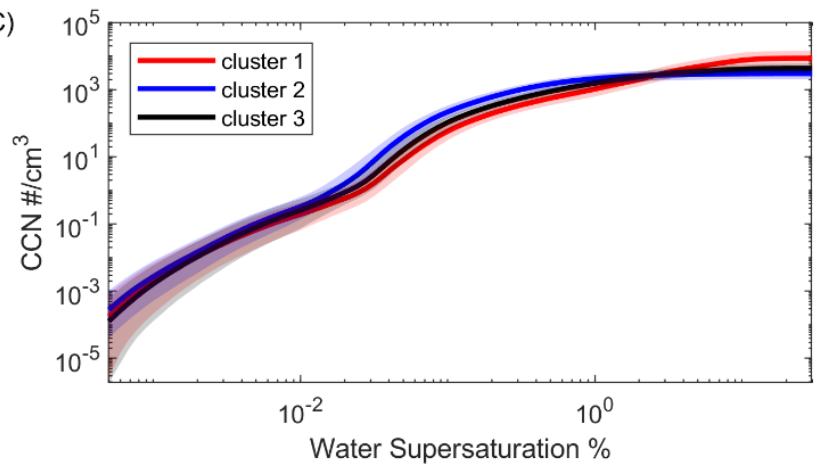

$\square$

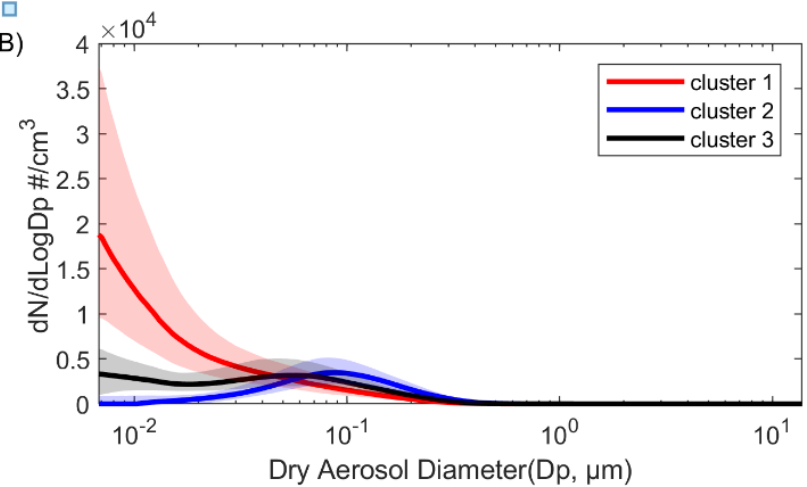

口

D)

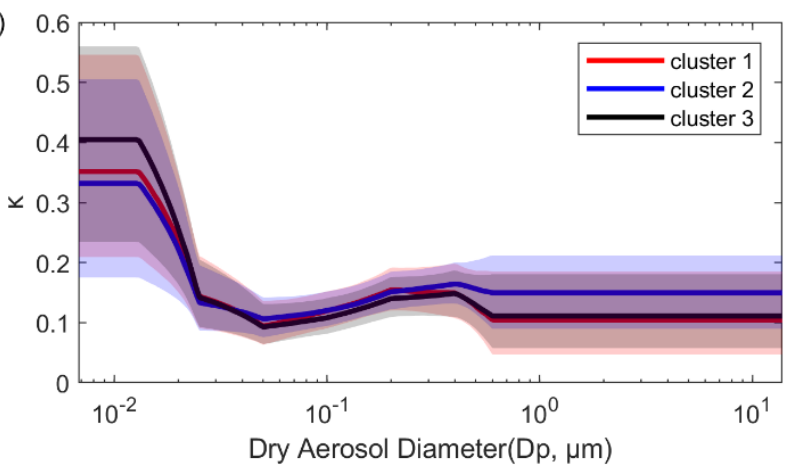

Figure 3: Clusters generated by k-means clustering procedure. Cluster centres are shown by solid lines, with shaded region representing $25^{\text {th }}$ and $75^{\text {th }}$ percentile bounds for spectra associated with each cluster. Panels A) and C) show cluster CCN spectra, panel B) shows cluster size distributions, and panel D) shows cluster hygroscopicity parameter $\kappa$.

Clustering is carried out based on CCN spectra - that is, each spectrum was assigned to a cluster based on its shape and magnitude. Even though the clustering procedure had no direct information about particle number size distributions, the size distributions associated with each cluster are well resolved (Fig. 3b). On the other hand, hygroscopicity parameter distributions are similar for all three clusters (Fig. 3d). This indicates that particle number size distributions have a greater influence on the resulting CCN spectra than do $\kappa$ distributions, consistent with other analyses (Patel and Jiang, 2021). As a result, estimates of $\mathrm{CCN}$ spectra using size distribution data and either estimated or median $\kappa$ values are expected to be reasonable approximations, although deviations of approximate $\mathrm{CCN}$ spectra from observed CCN spectra can still be quite large for any given timepoint. We estimate the error introduced using a "median $\kappa$ " to compute CCN spectra, as follows. For this "median $\kappa$ " estimate, we calculated a median $\kappa$ value based on the entire data set, then used this median value in combination with all individual size distributions to generate estimated CCN spectra. These are then compared to the CCN spectrum products (using concurrent $\kappa$ and size distribution data) to calculate error estimates, with the results shown in Figure 4. Estimates are generally least reliable for lower supersaturations, with estimates below $0.2 \% S S_{w}$ having a $95 \%$ confidence interval broader than $\pm 50 \%$ of the estimated value. Therefore, caution should be taken interpreting estimated CCN spectra in this low $S S_{w}$ region. This result additionally highlights the uncertainty in our data product below about $0.03 \% S S_{w}$. In this region, particles significantly larger 8 
than $600 \mathrm{~nm}$ are expected to activate but we do not have accurate $\kappa$ measurements for these sizes, as discussed in Section 2. Above $0.2 \% S S_{w}$, the "median $\kappa$ " estimate works quite well, with uncertainties decreasing as $S S_{w}$ increases. This region of the spectrum is likely a good candidate for generation of CCN spectra from observations of particle distributions where highquality $\kappa$ measurements are available for only a limited time period. It is important to note that this approach will only work for accurate number size distribution data extending to diameters larger than $500 \mathrm{~nm}$. For distributions ending at $500 \mathrm{~nm}$, many CCN activating at or below $\sim 0.2 \% S S_{w}$ will not be directly counted, and due to the cumulative nature of the distributions, this gap can introduce large errors for all $S S_{w}$ values. We expect this median $\kappa$ estimation method to be especially applicable for the SGP site and similar environments, but it may apply elsewhere as well.

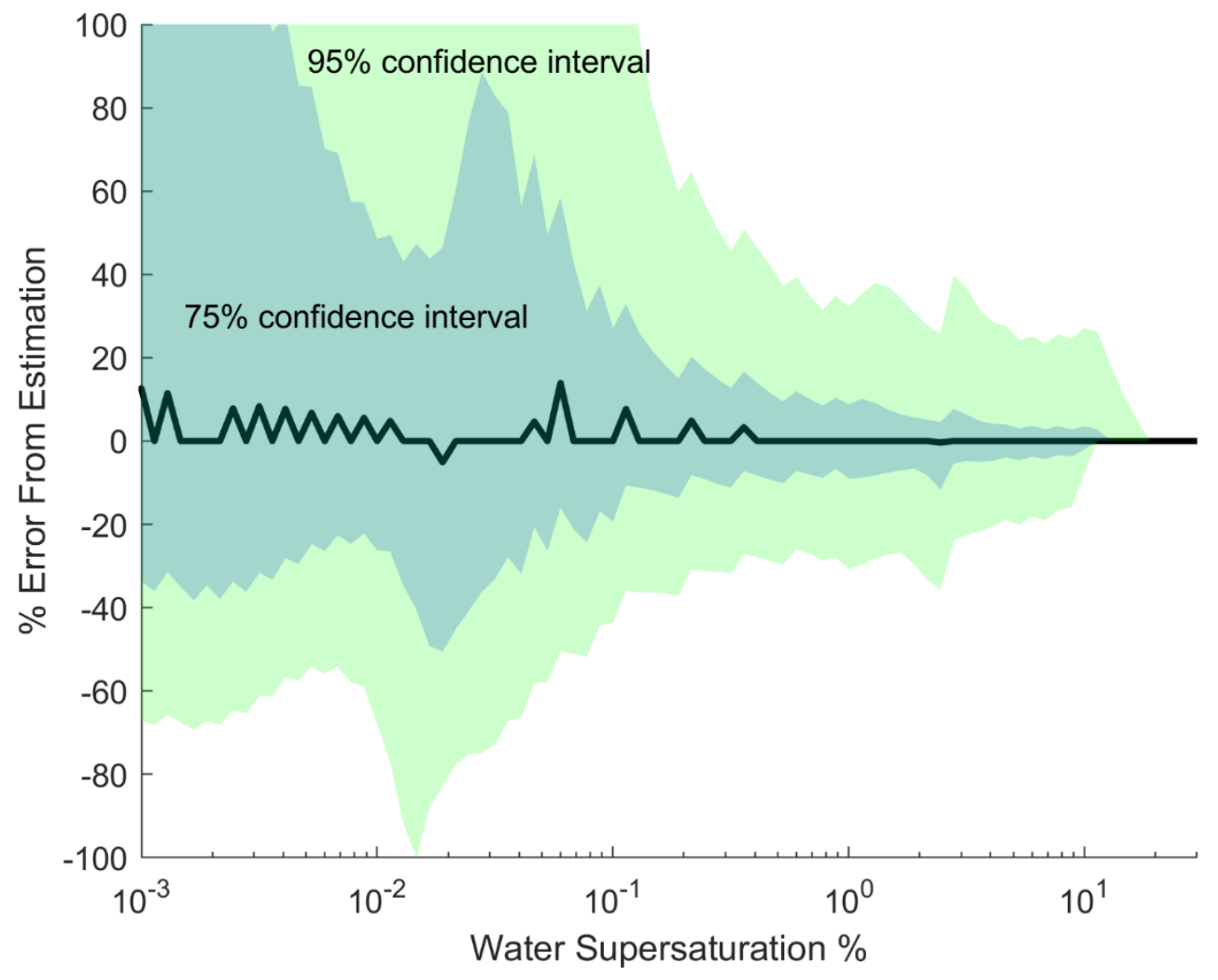

Figure 4: Error estimation of CCN product constructed from median hygroscopicity data. The black line depicts the median error, while the dark and light green shaded regions depict 75 and $95 \%$ confidence intervals, respectively.

The clustering of CCN spectra into distinct groups highlights the contributions from particle number size distributions as

195 the distinguishing factor. This analysis does not suggest that $\kappa$ distributions for small particles are invariant, random, or unimportant, only that their contribution to a final $\mathrm{CCN}$ spectrum is small compared to the contribution of the particle number 
size distribution. Mahish and Collins (2017) provide a more complete analysis of the $\kappa$ measurements at SGP during this time period, which is consistent with the data we use here.

Because of the factors discussed above, the different CCN clusters represent different characteristic particle number size

distributions. Cluster 1 represents cases where the nucleation mode particles, associated with NPF events, dominate the number size distributions. Cluster 1 also has lower absolute number concentrations of accumulation mode particles than found in the other clusters. Cluster 2 represents the opposite case - the absence of small particles and higher accumulation mode number concentrations combined with a shift of the accumulation mode to larger sizes. Cluster 3 is the intermediate case, with some nucleation mode particles and a substantial accumulation mode. The three clusters represent approximately equal portions of the total number of observations. Because these clusters represent three different scenarios quite well, we will use them to simplify further discussion. Cluster 1 will subsequently be referred to as the nucleation cluster, cluster 2 the accumulation cluster, and cluster 3 the intermediate cluster.

\subsubsection{Seasonal and Hourly Trends}

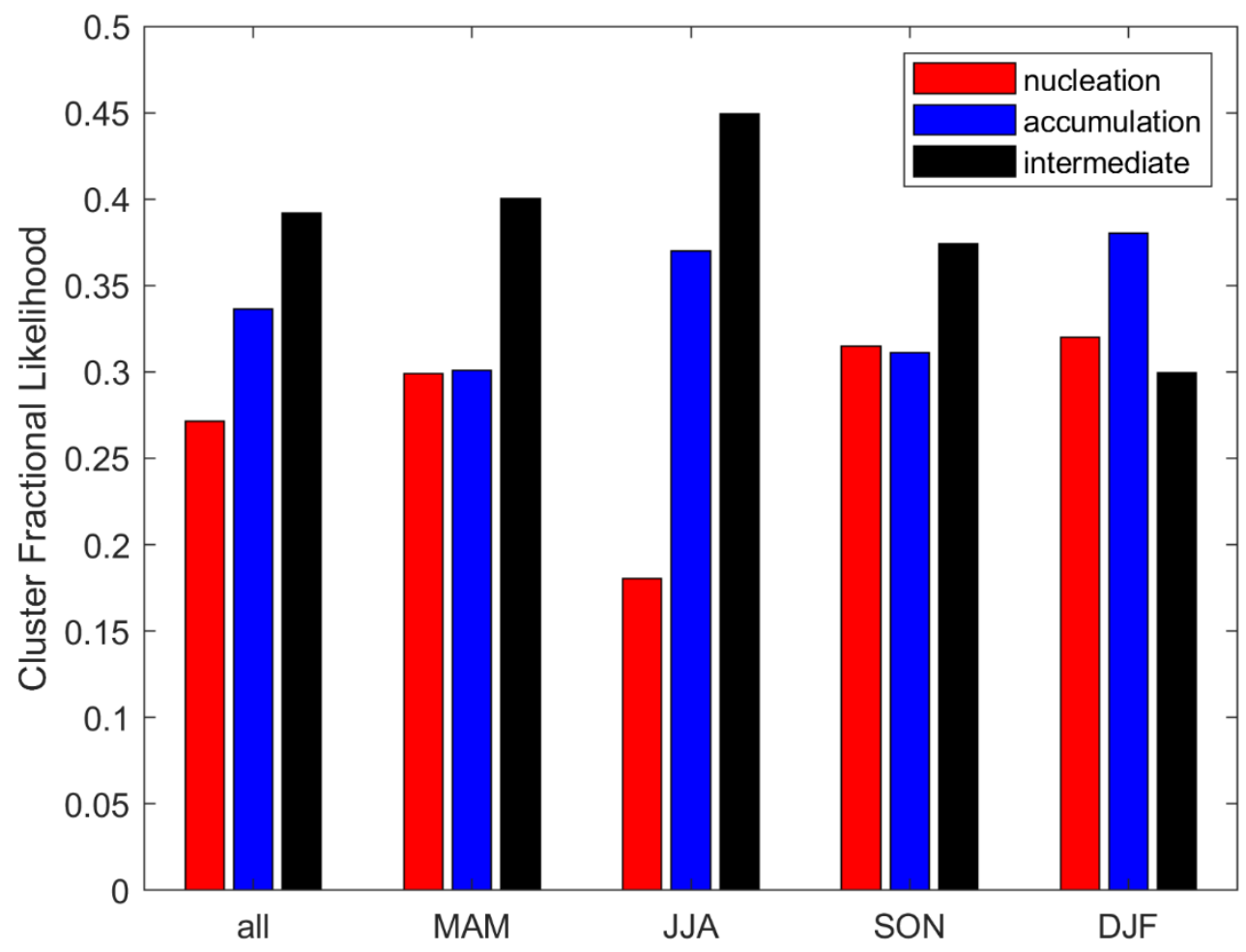

210 Figure 5: Cluster fractional likelihood for each season. 
The clustered data are examined for seasonal variations in particle and $\mathrm{CCN}$ characteristics. Cluster prevalence shows some seasonal dependence, although all clusters are still found a significant portion of the time for all seasons (Fig. 5). Summer (JJA) and winter (DJF) seasons show the highest prevalence of accumulation clusters, but significant differences in fractions of the intermediate and nucleation clusters. Summer has the highest prevalence of the intermediate clusters, while winter has 215 the highest prevalence of nucleation clusters. This suggests that during the summer significant particle concentrations are more likely to coexist in both the accumulation and nucleation modes, or perhaps that growth of nucleation mode particles to larger sizes (i.e., transfer of particles to the accumulation mode) is more likely to occur. These trends are not obvious from looking at seasonal particle data alone (Marinescu et al., 2019). An important consideration for reconciling seasonal particle data and seasonal cluster trends is the fact that the distributions within a cluster will have seasonal dependence as well, as shown in Figure $\mathrm{C} 1$.

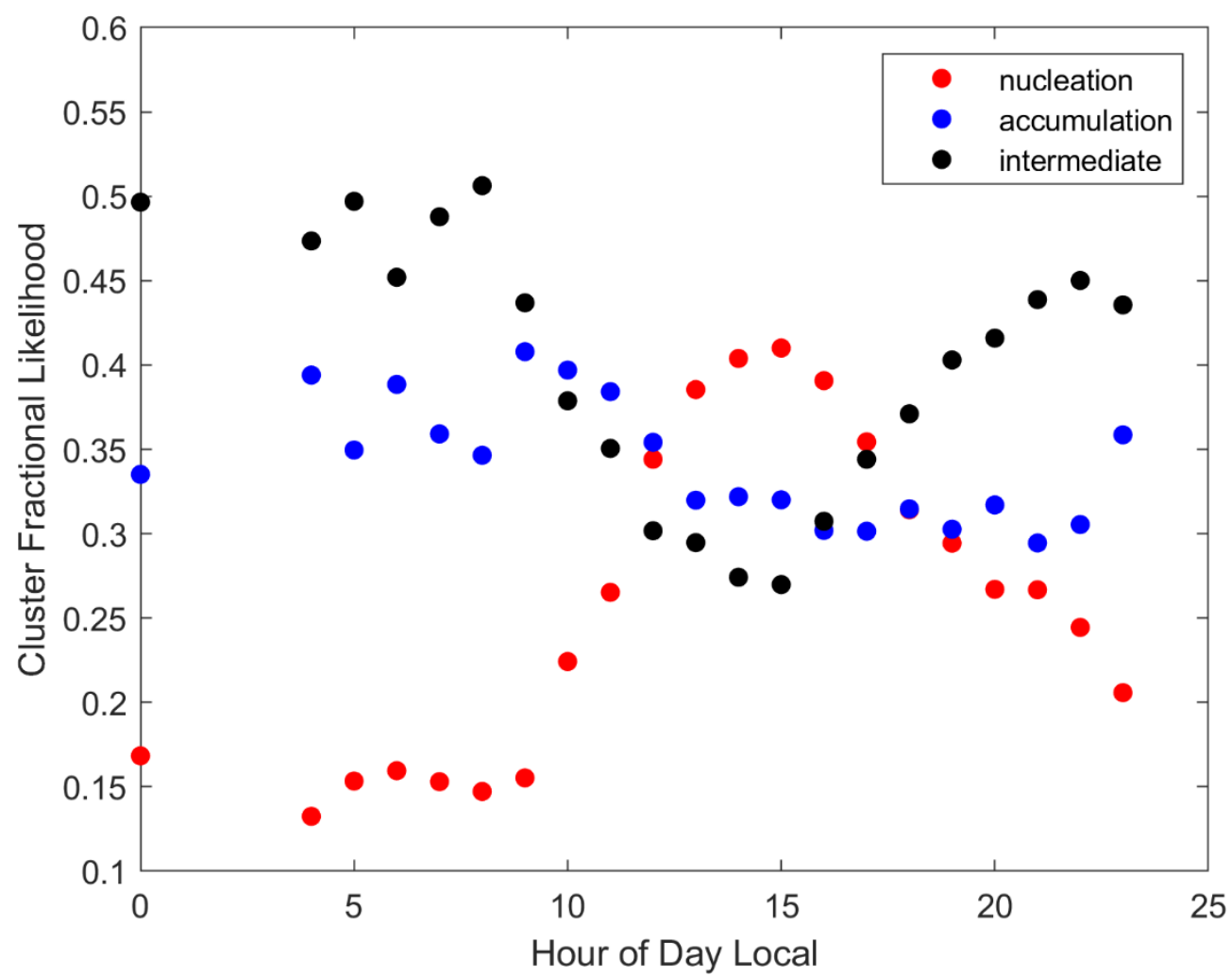

Figure 6: Cluster fractional likelihood for each local time of day.

Figure 6 depicts how cluster prevalence changes as a function of the time of day. Nucleation mode clusters are most common during daylight hours, with intermediate clusters most likely at night. Interestingly, accumulation clusters show the least time-dependence over the course of the day. Analysis of CCN concentrations at several supersaturations, as shown in 
Figure 7, shows no hourly dependence in median values at $1 \% S S_{w}$ or lower. At $10 \% S S_{w}$, the hourly trend in CCN is similar to the hourly trend in the nucleation cluster. These data combined suggest that the hourly changes that occur are due to addition of nucleation mode particles, rather than changes in the particle concentrations of other modes. Seasonal variability in the nucleation mode time-of-day dependence is shown in Figure $\mathrm{C} 1$ and reflects the same overall time dependence within a day, alongside seasonal changes shown in Figure 5.
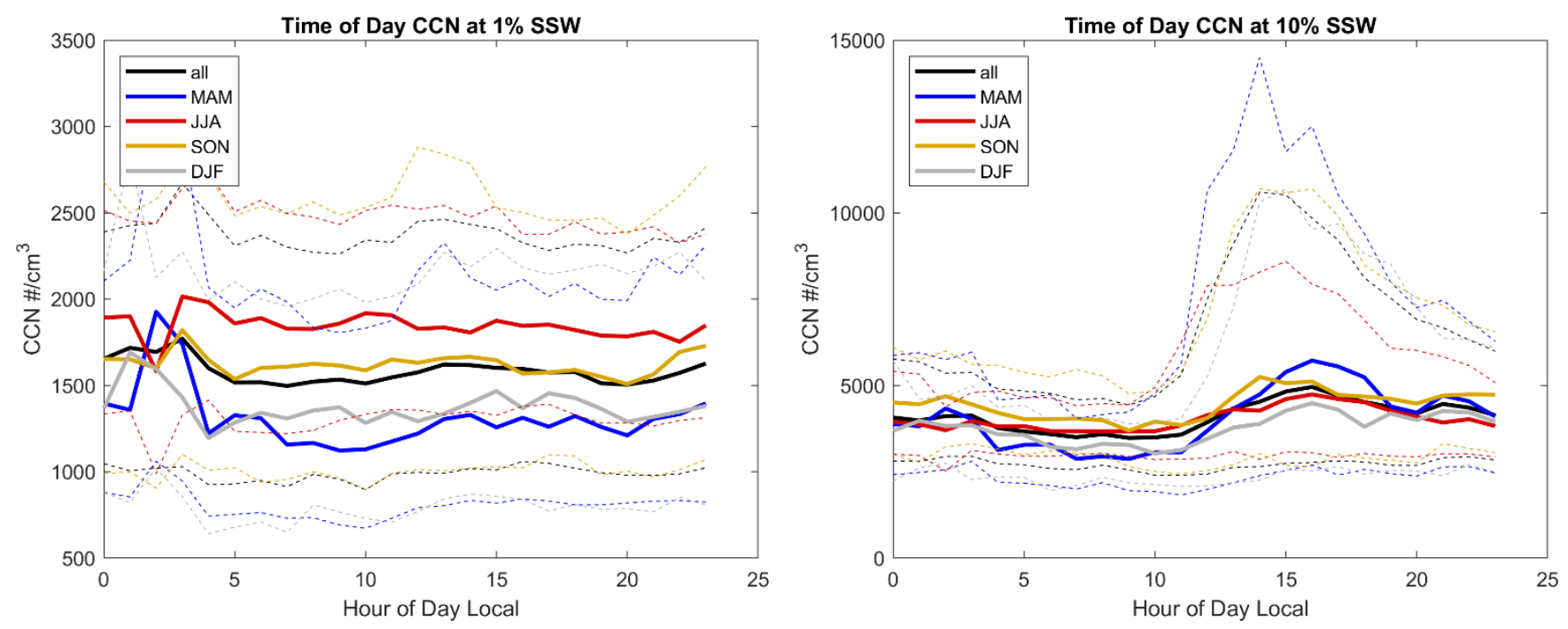

Figure 7: Median (solid lines) and $75 \%$ confidence intervals (dotted lines) seasonally averaged CCN concentrations at $1 \%$ (left) and $10 \%$ (right) $S S_{w}$, as a function of local time of day.

Another way to examine seasonal changes is through comparison of occurrence probabilities at a single supersaturation, as shown in Figure 8. Figure 8 shows this information in two similar ways: the skewed log-normal fits from Section 3.1 .1 and Appendix B, and more traditional box plots. Both methods of parameterizing the data require the same number of parameters ( 3 coefficients, a zero fraction and a correlation coefficient for the fit; 5 points for the box and whiskers), but the fits convey more information. Seasonal differences are somewhat obscured by the box plot, but for cumulative CCN active at $1 \%$ supersaturation, a clear difference between summer months and the rest of the year is observed with the fits, where $\mathrm{CCN}$ concentrations are more tightly grouped at higher values in the summer. Fit parameters for all supersaturations and seasons can be found in the Supporting File CCN_fit_coeffs.txt. The fits derived for cumulative CCN active at high supersaturations ( $1 \%$ and higher) are relevant to cases of deep convection, whereas those derived for very low supersaturations (below $0.1 \%$ ) may be helpful for estimates of the abundances of particles in special populations such as giant CCN. 

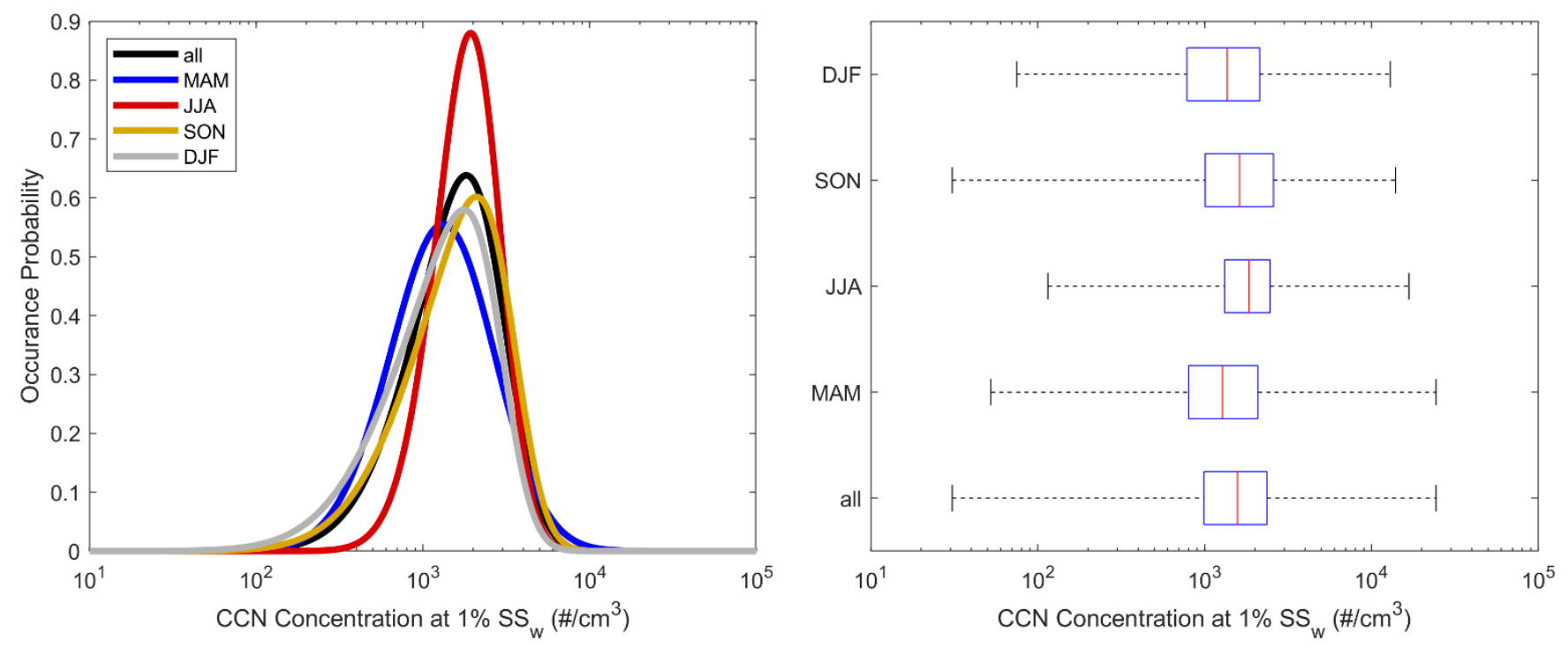

Figure 8: Distributions of $\mathrm{CCN}$ concentrations at $1 \% S S_{w}$ separated seasonally using skewed log-normal fits (left) and box plots (right).

\subsection{Time Evolution of Clusters}

Our large data set allows for additional statistical analysis to examine evolution of CCN spectra over time. This gives insight into underlying processes that are obscured when examining single cases or shorter data periods. Figure 9 shows the evolution of cluster classifications over time, examining all clusters starting in a given classification (nucleation, accumulation or intermediate). Cluster classification changes for all three clusters on the timescale of hours. Nucleation clusters are most likely to transition to intermediate clusters, rather than going directly to accumulation clusters. This could be through any or all of: growth of nucleation mode aerosol into larger sizes, coagulation scavenging/deposition/evaporation induced loss of the nucleation mode, and changes in air mass. Similarly, accumulation mode clusters are also more readily transitioned to intermediate clusters than to nucleation clusters. The role of intermediate clusters as the pathway of conversion between accumulation and nucleation clusters is further reinforced by the fact that they are equally likely to transition to either cluster type. In terms outside of the clustering perspective, it appears most likely that transitions from aerosol distributions dominated by nucleation mode particles to ones dominated by accumulation mode particles (or vice versa) occur smoothly through intermediate cases where both modes are of similar magnitude, rather than abruptly. However, the analysis cannot distinguish the specific role of meteorology in these transitions. 

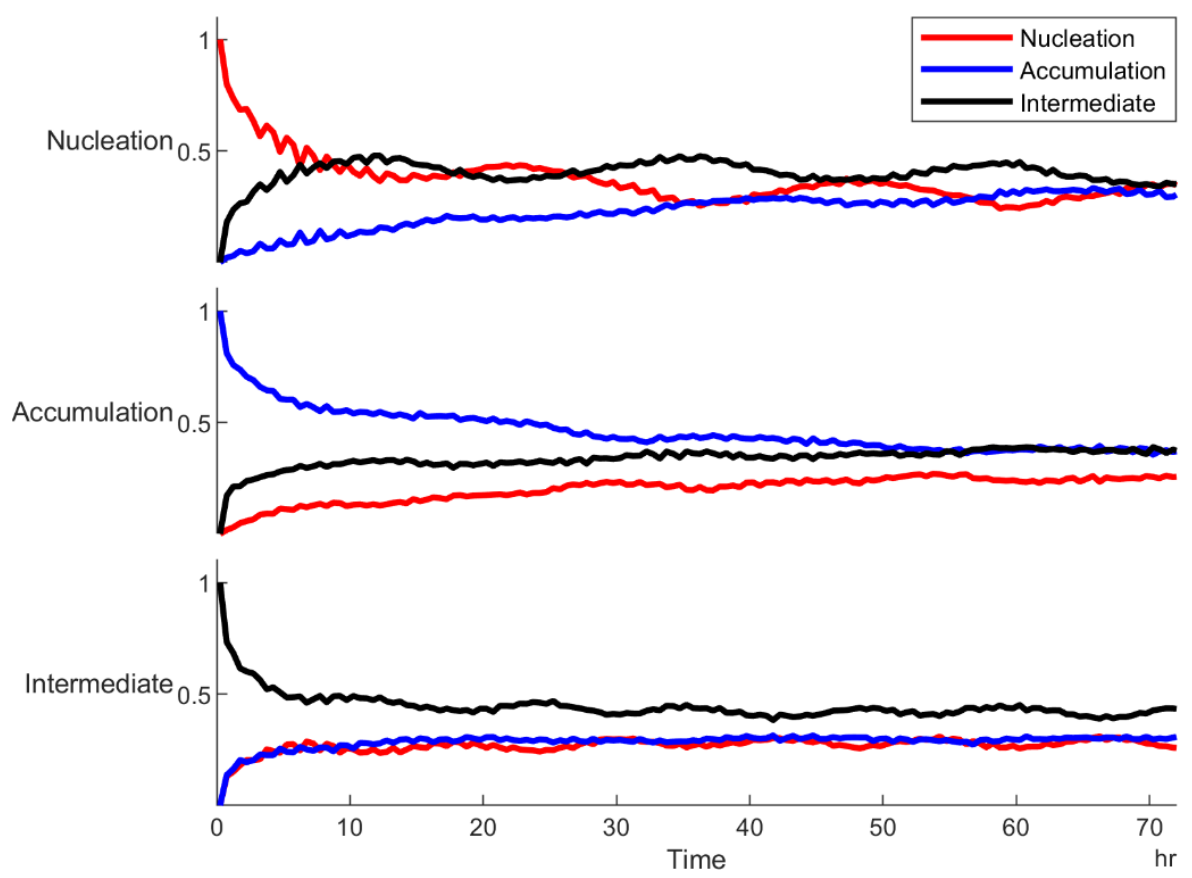

Figure 9: Cluster evolution over time (hours after appearance of cluster). $\mathrm{Y}$ axis for each plot shows the likelihood of the cluster transitioning to a new cluster type after a specified time, $X$ axis, has elapsed. The first collection of traces labelled "Nucleation" shows evolution of nucleation cluster spectra into the other categories, with the middle collection depicting evolution of accumulation cluster initial states and the bottom showing evolution of intermediate cluster initial states. Traces indicate final states (after the specified time lag) of the nucleation cluster (red), accumulation cluster (blue) or intermediate cluster (black).

There is some periodicity observed in the cluster evolution, which we examine more closely alongside fluctuations in CCN number concentrations using autocorrelations. Autocorrelation coefficients are calculated for several different $S S_{w}$ conditions, shown in Figure 10, using the methods described in Appendix D. Autocorrelation coefficients can be interpreted similarly to other correlation coefficients - they describe that portion of the variance that can be explained by the observation at a previous timepoint. The higher the value of an autocorrelation coefficient, the more stable that quantity is over time, so that an autocorrelation coefficient of 1 implies no change in state at a specified time lag, while 0 implies that a previous data point (separated by the specified time lag) has no influence on a current one. From Figure 10, a great deal of variability is observed in the first several hours of the computed time lags, which is an unexpected finding. Autocorrelation coefficients are expected to be highest for the first several timepoints, but the oscillating nature of these points implies aerosol processes with some periodicity in the 2-3 hour range. Natural processes that might produce such variability throughout the day and over all seasons seem unlikely, so the oscillation may be an artefact, for example, introduced by sampling schedules. We apply a bi- 
exponential fit as an approximate way to smooth the data for time lags of up to twelve hours, removing the effect of these oscillations. Single exponential fits are poor approximations of the shape of the autocorrelation functions for 0.1 and $1 \% S S_{w}$ cases. Because a single decay pathway is expected to produce relatively consistent decay rates, the appearance of multiexponential decays suggests multiple decay pathways. In this case decay pathways for autocorrelation can be interpreted as pathways for changes in $\mathrm{CCN}$ number concentrations.

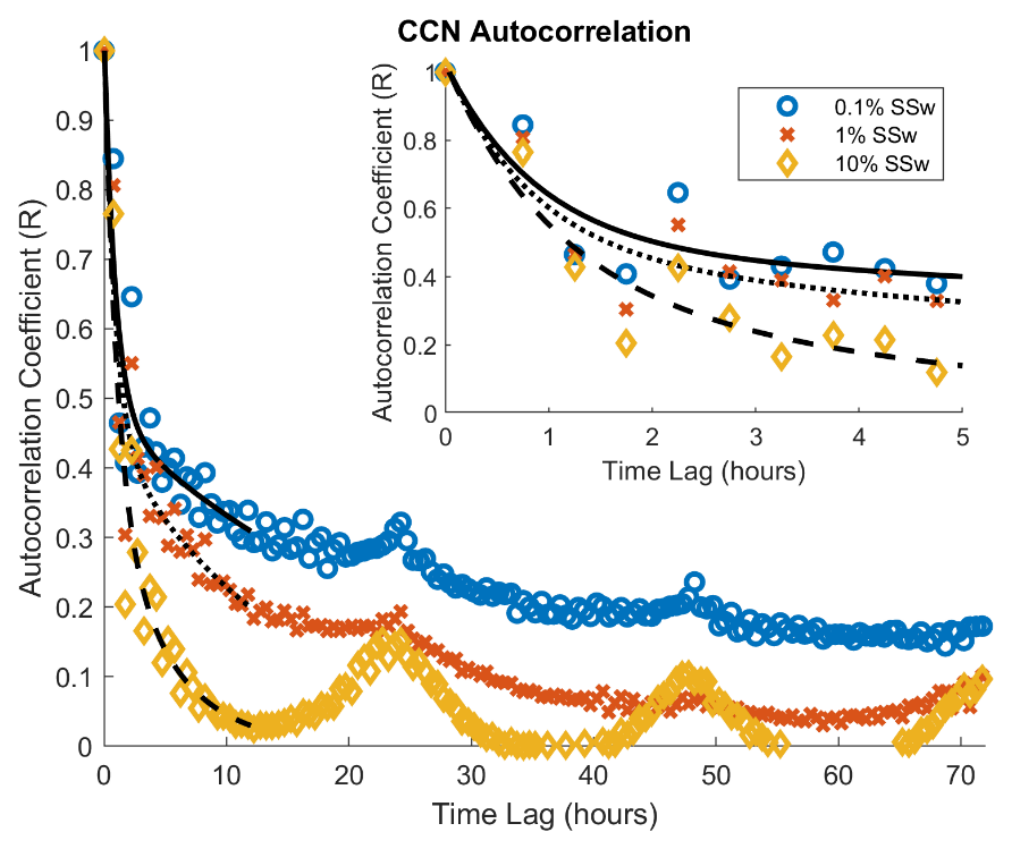

Figure 10: Autocorrelation functions for $\mathrm{CCN}$ number concentrations at variable $S S_{w}$. Bi-exponential fits are shown for the first 12 hours of lag time for each $S S_{w}$, with solid, dotted, and dashed lines corresponding with $0.1 \%, 1 \%$, and $10 \% S S_{w}$, respectively.

Autocorrelation decays much more quickly for larger $(10 \%) S S_{w}$, with greatly increased values appearing at 24-hour intervals. At higher $S S_{w}$, the $\mathrm{CCN}$ number concentration is often dominated by the smallest particles, associated with the nucleation mode. This interpretation fits well with the autocorrelation data, which indicate short-lived events tied to diurnal cycles. At moderate $(1 \%)$ and low $(0.1 \%) S S_{w}$, there is a pronounced fast initial decay in autocorrelation, followed by a period of slower decay. Fit constants are described in appendix D and shown in Table D1. The fast initial decay rate is comparable (within the large uncertainties, Table D1) for low and moderate $S S_{w}$ cases, but the slow decay rate is significantly slower for the low $S S_{w}$ case. The diurnal peak (at 24-hour time lag) is also significantly weaker for the lowest $S S_{w}$ case, suggesting that there is less variability in these lowest $S S_{w} \mathrm{CCN}$ observations, as compared to higher $S S_{w}$. This is consistent with that fact that number concentrations at larger particle sizes are much less variable than those at smaller particle sizes (Marinescu et al., 2019). Previous work on AOD spatial and temporal autocorrelation at the SGP site (Alexandrov et al., 2004) and elsewhere (Anderson et al., 2003) suggest that the fast decay can be attributed to 3D micro-scale turbulent fluctuations, while the slow 15 
decay is due to 2D large-scale turbulence. The aerosol data used here are obtained only at the surface, to the influence of the three-dimensional nature of the atmosphere may be present but cannot be distinguished. Given the role of NPF events, there is also potentially a chemical (non-turbulence-driven) source of variability for CCN, contributing to autocorrelation decay for $\mathrm{CCN}$ concentrations at high $S S_{w}$. Ultimately these data illustrate that CCN spectra change rapidly over 1-3 hour timescales, with some conservation at longer timescales for the lower end of the supersaturation range. The granularity of our data (in 45-minute increments) makes it somewhat difficult to resolve the exact timescales, but it is clear that the period of rapid change is in the 1-3 hour range. The role of variability in $\mathrm{CCN}$ concentration is something that should be considered in modelling studies that focus on the impacts of aerosol, especially those that use fixed concentrations of aerosol particles or those that do not capture the comprehensive processes that cause aerosol concentrations and properties to evolve. For example, using fixed CCN concentrations for a given short-term ( $<2$ hour) simulation of shallow clouds (i.e., lower supersaturations) is more justifiable than for a longer-term simulation of the development of deep convective clouds (i.e., higher supersaturations), based on the faster autocorrelation decay rates of CCN at higher supersaturations. The autocorrelation results can also help define the time scales for data assimilation to ensure models are updated frequently enough to allow for accurate simulations.

\section{Conclusions}

We have developed, described and examined a long term CCN spectrum data product for the SGP site in Oklahoma. The data product builds on merged number size distributions (Marinescu et al., 2019) and hygroscopicity measurements (Mahish and Collins, 2017) to create a best estimate of CCN spectra across a wide supersaturation range from $\sim 0.0001 \%$ to $30 \%$. It has been generated and verified by combining high quality data from eight different instruments. It has $\sim 45$ minute temporal resolution across five years of data, from 2009 to 2013, which has allowed for analyses not normally possible for smaller data sets.

We have determined that skewed log-normal distributions provide excellent fits to occurrence probabilities of $\mathrm{CCN}$ concentrations at any given supersaturation, as well as to occurrence probabilities of a wide range of other aerosol quantities. These types of distributions have been observed for AOD measurements previously, but not widely used. They provide more information than traditional box plots, while requiring a comparable number of parameters. For established occurrence distribution shapes, shorter timescale measurements could likely take advantage of these fit parameters to fill in data gaps to estimate data over longer periods. They also serve as useful inputs to models that include the expected variability in input parameters in model predictions.

CCN spectra are controlled primarily by particle number size distributions, especially at larger $S S_{w}$ values (above $\sim 0.2 \%$ ). In this high $S S_{w}$ region of the spectrum, it appears possible to generate estimated CCN spectra using only median $\kappa$ values, rather than concurrent measurements of $\kappa$ and size distribution. However, this estimation relies on accurate size distribution 16 
data that extend beyond $500 \mathrm{~nm}$. Approximations of uncertainties introduced by this "median $\kappa$ " estimation have been investigated and should hold for data from the SGP site during different time periods. This estimation method is also likely applicable for other sites, especially in similar environments, but is beyond the scope of this analysis.

Clustering analysis also highlights number size distributions as the driving force behind changes in CCN spectra. Three distinct clusters are found for cases dominated by nucleation mode particles, accumulation mode particles, or similar amounts of each. These are analyzed seasonally and hourly, finding all clusters in significant quantities across all seasons and times. Intermediate clusters are more likely during summer months, while accumulation clusters are abundant in the winter. Fall and spring appear similar in this view, falling between summer and winter. Nucleation mode clusters are most likely during daylight hours, corresponding with decreased intermediate clusters but nearly invariant accumulation clusters.

Time evolutions are examined in this data set to try to understand the dynamics of CCN spectra. Analysis of transitions between clusters reveals that the most likely path is for nucleation and accumulation mode clusters to transition to an intermediate cluster first, rather than direct transitions occurring between the two. Autocorrelation analyses probe the evolution of a given CCN $S S_{w}$ bin over time. A relatively quick decay is found for all $S S_{w}$ values, with the bulk of correlation decaying in several hours, indicating that relatively large changes in CCN spectra can be expected over that time period. An additional slow decay is observed for smaller $S S_{w}$ values, indicating that the CCN number is better conserved at longer timescales ( $>2-3$ hours) in lower $S S_{w}$ regions of the CCN spectrum, corresponding to particles in the coarse mode.

\section{Appendix A: Merging of Distributions and CCN Spectra}

The initial number size distributions used in this work are generated from a combination of scanning mobility particle sizer (SMPS), aerodynamic particle sizer (APS), and condensation particle counter (CPC) data, as described previously by Marinescu et al., (2019), which are available in the DOE ARM archive (Marinescu and Levin, 2019). This initial dataset, here referred to as size distribution (SD) 1 , is processed to take into account additional instrument data utilizing humidified tandem differential mobility analyzer (HTDMA), CCN counter (CCNC), nephelometer, and aerosol chemical speciation mass spectrometer (ACSM) instrument data as outlined in Figure A1. SD1 data is available in approximately 45 minute time intervals, where additional instrument data are available in higher time resolution, and are subsequently averaged over the time period of SD1 data for comparison. In order to compare to CCNC measurements, SD1 must be converted to CCN spectra using the hygroscopicity parameter $(\kappa)$ that is derived from HTDMA measurements. 


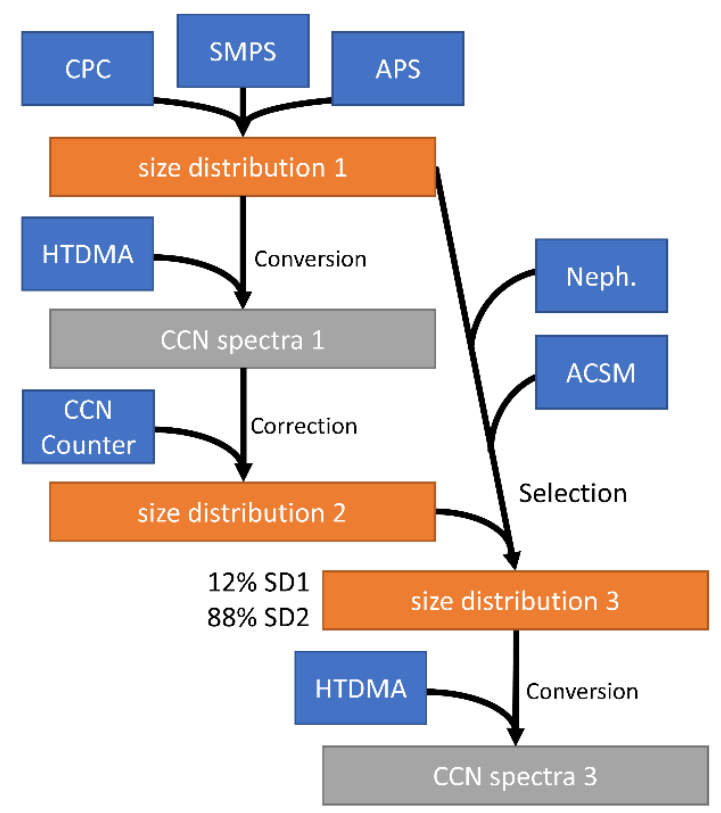

Figure A1: Flowchart illustrating processing of data to various distributions, with input instrument data shown in blue boxes, number size distributions in orange, and CCN spectra in grey.

Size resolved aerosol hygroscopicity was measured with a HTDMA (Collins, 2010), which first selected dried, monodisperse aerosol at seven diameters and then exposed them to a humidified (relative humidity, RH 90\%) growth region. The humidified aerosol number size distribution was then measured and the change in particle diameter between the selected dry particle diameter $\left(D_{p}\right)$ and the resulting humidified size $\left(D_{w}\right)$ is termed the growth factor $\left(\mathrm{GF}=D_{w} / D_{p}\right)$. An example of HTDMA measured growth factor distributions is shown in Figure A2. The orange lines indicate the selected dry size for each GF measurement. The top part of the figure shows the number size distribution measured by the SMPS at the same time. 


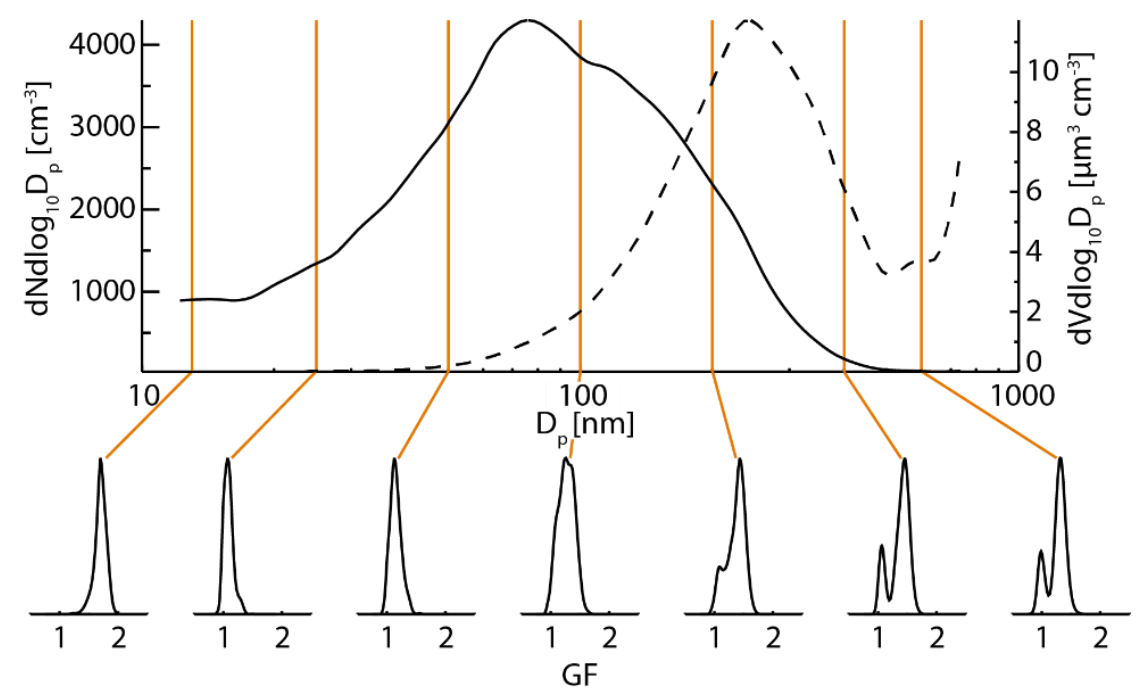

Figure A2: Example aerosol number distribution (solid line, upper plot) and growth factor (at 90\% RH) distributions (lower plots) measured by an HTDMA. The dashed line in the upper plot is the corresponding volume distribution.

These GF data at a given RH (properly written e.g. GF(90\%), but here abbreviated to GF for convenience) can be used to calculate the hygroscopicity of the particles as expressed via the hygroscopicity parameter, $\kappa$ (Carrico et al., 2010; Petters and

Kreidenweis, 2007):

$$
\kappa=\frac{\left(G F^{3}-1\right)\left(1-a_{w}\right)}{a_{w}}
$$

where

$$
a_{w}=\frac{R H}{\exp \left(\frac{4 \sigma_{w} M_{w}}{\rho_{w} R T D_{d} G F(R H)}\right)}
$$

and $\sigma_{w}, M_{w}$ and $\rho_{w}$ are the surface tension, molecular weight, and density of water, respectively, $\mathrm{T}$ is the absolute temperature, and $\mathrm{R}$ is the ideal gas constant. After calculating $\kappa$ distributions from each measured GF distribution at the diameters selected by the HTDMA we averaged $\kappa$ for each selected $D_{d}$ and linearly interpolated between selected sizes to generate a continuous distribution of aerosol hygroscopicity across the entire number size distribution.

CCN spectra were generated using these $\kappa$ values, derived from the HTDMA growth factor data described above using either the SD1 (initial) or SD3 (final) size distributions (described below). For each time period with concurrent $\kappa$ and size distribution data, critical $S S_{w}$ was calculated for each size bin. This was accomplished using Eq. 1 assuming the following 
constant conditions: temperature of $25{ }^{\circ} \mathrm{C}$, water density of $1 \mathrm{~g} / \mathrm{mL}$, and surface tension of $72 \mathrm{~mJ} / \mathrm{m}^{2}$. This calculation is accomplished numerically, by calculating water $S S_{w}$ for a logarithmically-spaced array of wet diameters, with the largest $S S_{w}$ chosen as the critical value. Calculated errors from this method were less than $1 \%$ of the calculated values using this method (e.g. a $1 \%$ error in a $0.01 \% S S_{w}$ being $\pm 0.0001 \% S S_{w}$ ). A CCN spectrum was then generated by adding up activated particle populations at each $S S_{w}$ value, making no assumptions about the order particles activated in; smaller particles with higher $\kappa$ could activate before large particles with low $\kappa$ if appropriate.

In order to compare spectra between CCN spectrum 1 and measured CCNC values averaged across the same 45 -minute time intervals, interpolation of the CCNC data across the calculated $S S_{w}$ bins was performed using MATLAB's built in Piecewise Cubic Hermite Interpolating Polynomial (PCHIP) function for each CCNC spectrum. These data were used to create a distribution corrected for the CCNC data using a similar method to that described for the CPC corrections in SD1 (Marinescu et al., 2019). The only difference between the algorithm described in Appendix A of Marinescu et al. (2019) and the one used for generation of SD2 here occurs in step 3. We calculated a two-week rolling median percent difference between the CPC and SMPS + APS distribution and used this as a scaling factor across the entire distribution in this step. Times between 1200 and 1800 local are excluded from the rolling median since new particle formation events are common during those times and large differences between the SMPS+APS integrated number concentration and the CPC number concentration are expected. To generate SD2, the average difference between CPC and CCNC (in total particles and $S S_{w}$ specific CCN numbers, respectively) is used instead of solely CPC data. For example, if the comparison with the CPC suggested there should be 25\% more particles in the SMPS+APS number size distributions and the comparison with the CCNC suggested there should be $15 \%$ fewer particles in the SMPS+APS number size distributions, then the SMPS+APS number size distribution data are scaled up by $5 \%$ (the average of $+25 \%$ and $-15 \%$ ). The remaining steps described in (Marinescu et al., 2019) are performed unchanged on this distribution. The remaining steps in the algorithm can change the shape of the size distribution, so SD1 and SD2 are not simply scaled versions of each other. If no quality CCNC data are available for a given timepoint, the SD1 and SD2 spectra are identical.

The resulting SD2 was then compared to ACSM and nephelometer data to examine whether the CCNC correction was warranted. ACSM comparisons were accomplished by generating total particle mass concentration for each distribution assuming spherical particles and a density of $1.77 \mathrm{mg} / \mathrm{mL}$, which is that of ammonium sulfate. Additionally, the ACSM cut405 off of $10 \mu \mathrm{m}$ and the volume equivalent diameter (DeCarlo et al., 2004) were accounted for to produce calculated aerosol mass for comparison. The density chosen is within the region of best agreement between the ACSM and distribution data for both the SD1 and SD2 and is chosen for consistency with the nephelometer comparison. The nephelometer comparison was accomplished by generating single-particle scattering cross-sections for all size bins in the distributions assuming the optical properties of ammonium sulfate, which was again within the region of best agreement in Figure A3. Both of these comparisons 
410 produce excellent agreement for many time points, as shown in Figure A4. There was evidence of systematic bias for some time periods but the bias was relatively low for periods outside of 10-Mar-2011 through 1-Nov-2011 for the nephelometer data, which were not used in distribution selection, below.
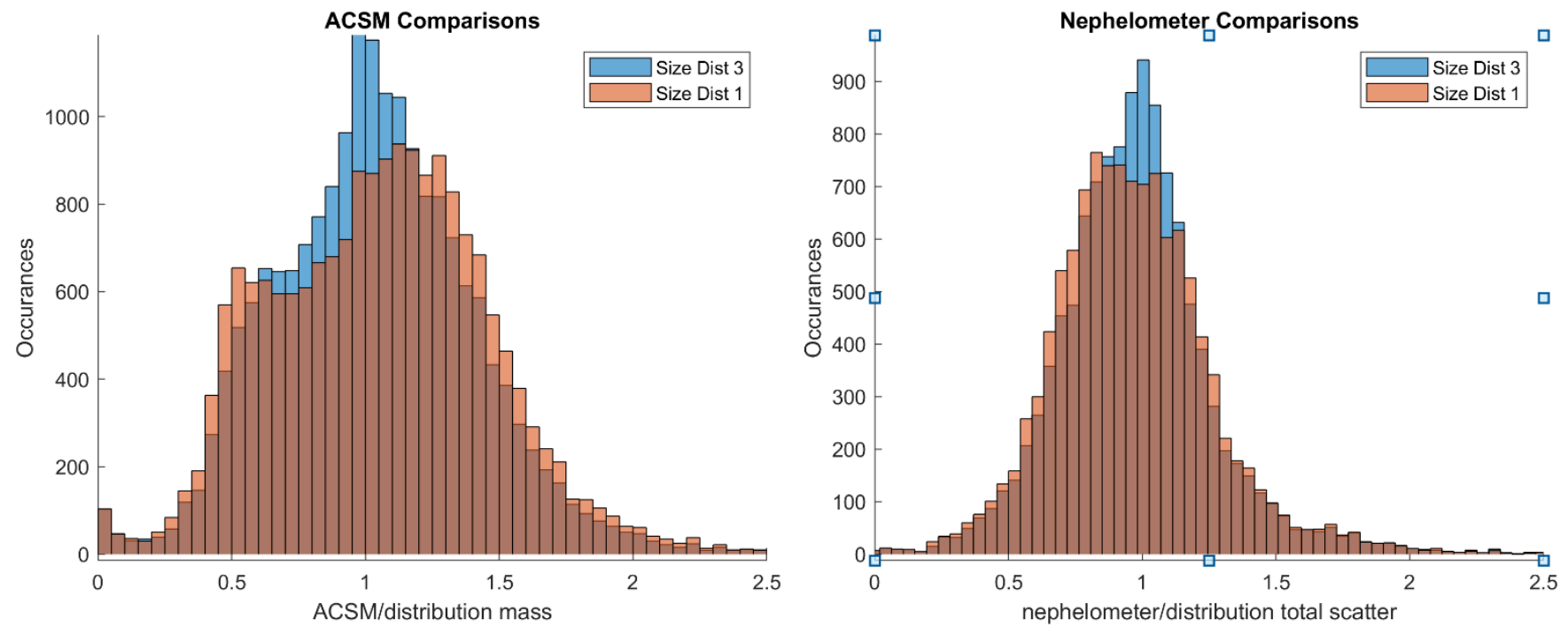

Figure A3: Statistical comparisons of the agreement between measured quantities (left, ACSM aerosol mass concentrations; right, show the comparisons as ratios, where a ratio of 1 indicates perfect agreement.

SD2 generally produced better agreement with the nephelometer and ACSM than SD1 did, although this was not true for all time periods. In order to construct a final data set including the nephelometer and ACSM comparisons, the ratios between distribution-calculated values and measured values were used to select between SD1 and SD2 at each point where data were available. Given the better general agreement for SD2, it was used as default if there were no ACSM or nephelometer data available, or if there was disagreement between the two instruments. Through this process, 4711 distributions were selected from SD1, and 16203 from SD2, with 19407 points defaulting to SD2. The resulting distribution, SD3 compared with ACSM and nephelometer measurements in Figure A3, is considered the final product distribution, and analyzed throughout the manuscript alongside the $\mathrm{CCN}$ spectra generated from it. 

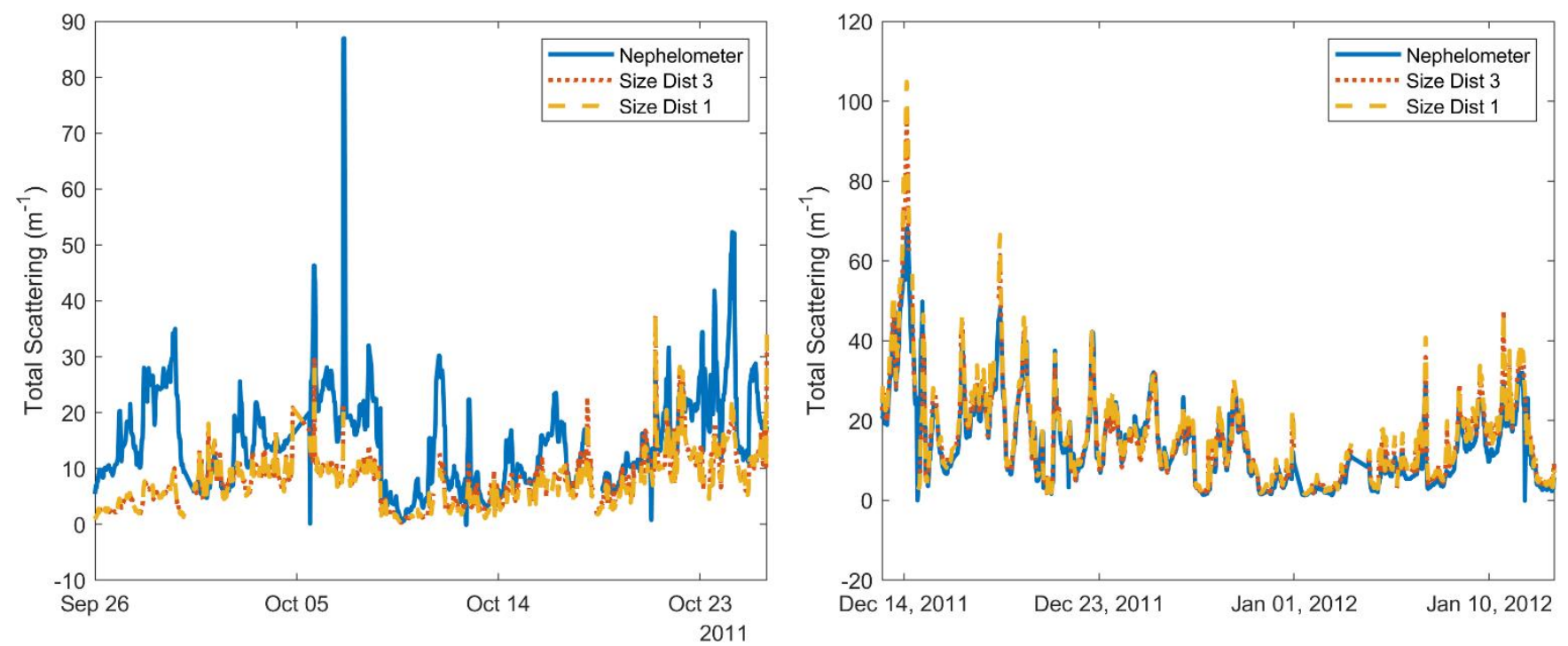

Figure A4: Examples of excluded (left) and included (right) nephelometer data (blue traces) compared to total scattering coefficients calculated from the indicated estimated number size distributions (SD1 or SD3).

\section{Appendix B: Skewed Log-Normal Fits}

The log-normal probability density function is defined as:

$$
\varphi(\ln x)=\frac{1}{\mathrm{x} \sigma \sqrt{2 \pi}} \exp \left(-\frac{(\ln x-\mu)^{2}}{2 \sigma^{2}}\right)
$$

Eq. 4

430 where $\mathrm{x}$ is a number concentration (bin), $\mu$ is the median value of $\log (\mathrm{x})$, and $\sigma^{2}$ is the variance in $\log (\mathrm{x})$. The $\log$-normal cumulative distribution function is defined as:

$$
\Phi(\ln x)=\frac{1}{2}+\frac{1}{2} \operatorname{erf}\left[\frac{\ln x-\mu}{\sigma \sqrt{2}}\right]
$$

where erf is the error function. A skewed log-normal probability density function can subsequently be defined as:

$$
f(\ln x)=2 \varphi(\ln x) \Phi(\alpha \ln x)
$$

Eq. 6

where $\alpha$ is a parameter representing the degree of skew, such that when $\alpha=0$, the log-normal distribution is recovered. When these functions are used to fit data, $\mu, \sigma$, and $\alpha$ are used as the fit parameters. 
Two issues arise using skewed log-normal fits. The first is that there is no closed-form expression for the median value of a skewed log-normal distribution. The median can of course still be evaluated numerically. The second is that $x$ values of zero cannot be represented within the distribution due to the logarithm. In this work, we address these issues by simply reporting median values and fractions of the data where zeros occur, alongside fit parameters. It should be noted $\mu$ is no longer the median value of $\log (\mathrm{x})$ for the skewed $\log$-normal distribution. This can be observed in the data in Supporting File 440 CCN_fit_coeffs.txt where $\mu$ decreases at high $S S_{w}$ while median value increases monotonically, as expected for a cumulative distribution. Fits are generally very good, as shown in Figure B1 for several different aerosol quantities. At very low $S S_{w}$, or for very large particle bins, the quality of the fits degrades due to the large amount of noise in the data. This noise occurs largely due to detection limits of the instruments involved at low particle concentrations (for very large particles). If concentrations are so low that particle detections are not guaranteed in the sampling period (45 minutes), a large amount of 445 shot noise is introduced. 

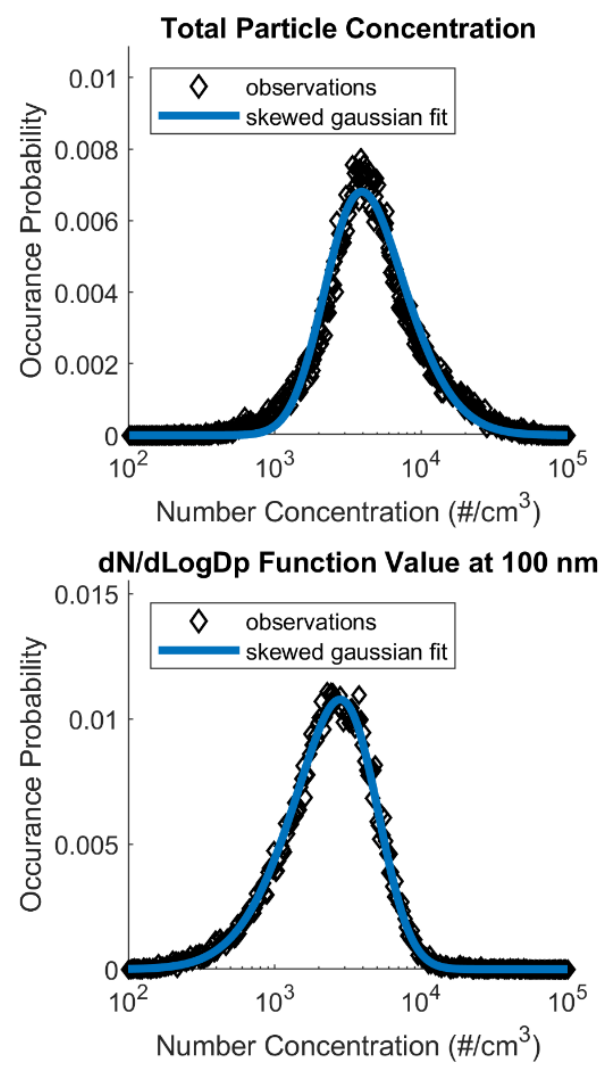

Cumulative $\mathrm{CCN}$ at $0.1 \% \mathrm{SSw}$

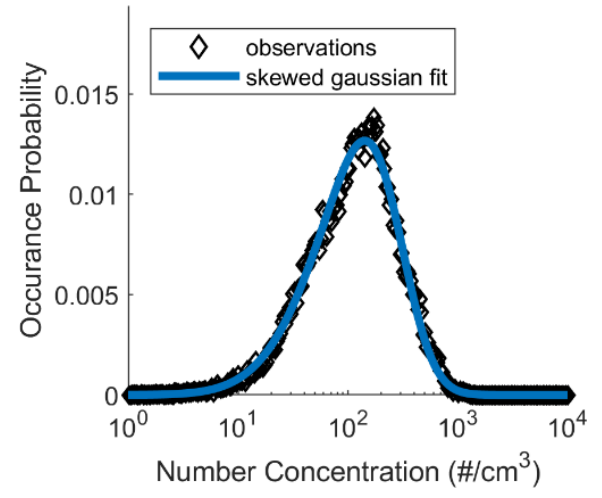

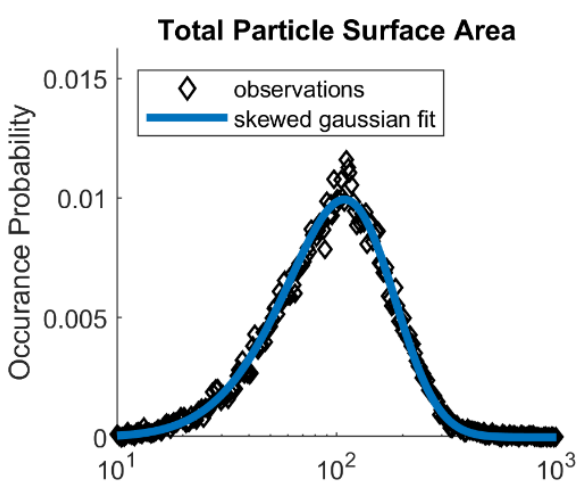

Surface Area Concentration $\left(\mathrm{m}^{2} / \mathrm{cm}^{3}\right)$

dN/dLogDp Function Value at 1000 nm

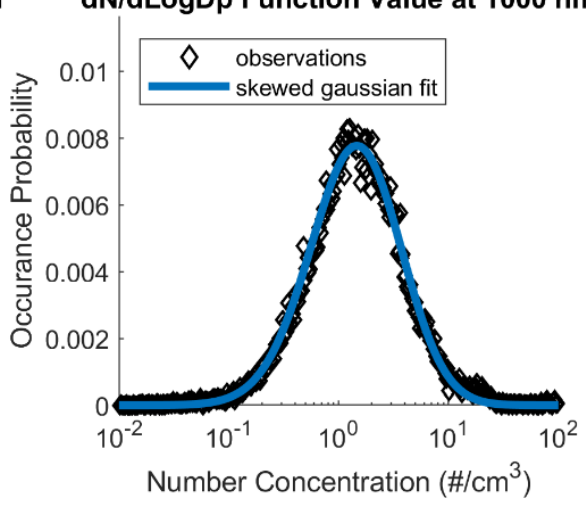

Cumulative $\mathrm{CCN}$ at $2.8 \% \mathrm{SSw}$

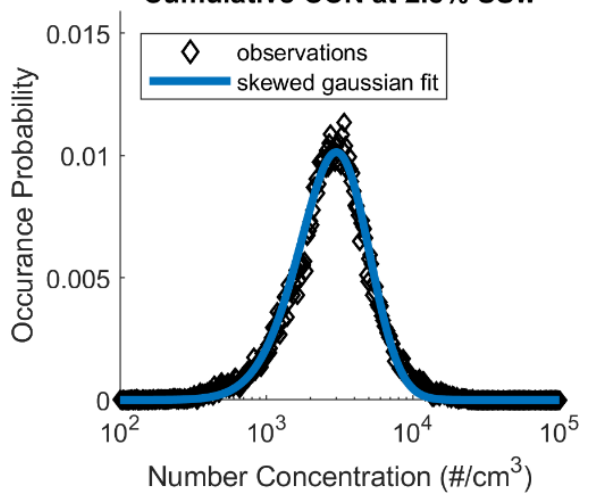

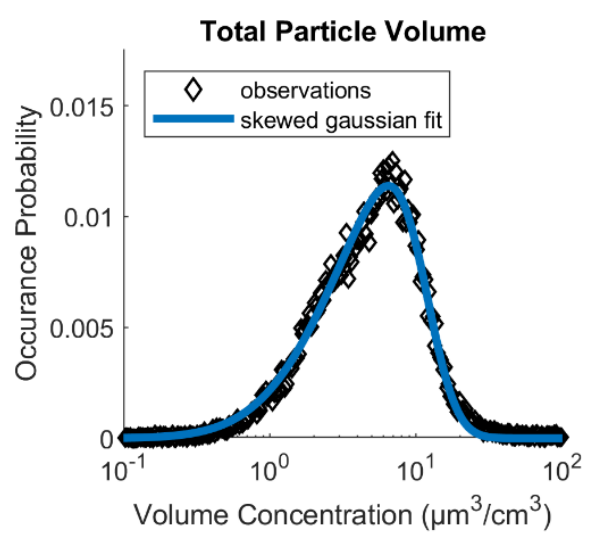

Cumulative $\mathrm{CCN}$ at $0.01 \% \mathrm{SSw}$

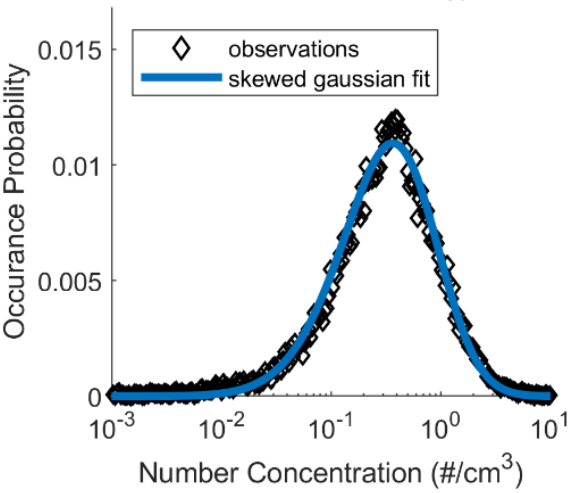

Cumulative $\mathrm{CCN}$ at $5.3 \% \mathrm{SSw}$

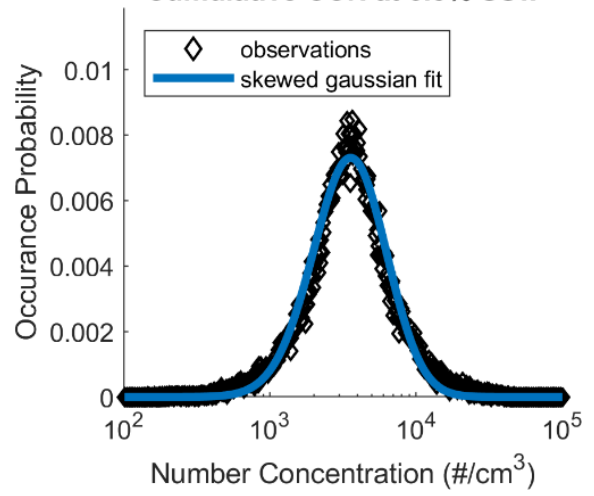

Figure B1: Examples of skewed log-normal fits to occurrence probabilities for aerosol metrics, as labelled: total particle number, total particle volume, $\mathrm{dN} / \mathrm{dLogDp}$ function at $100 \mathrm{~nm}, \mathrm{dN} / \mathrm{dLogDp}$ function at $1000 \mathrm{~nm}$, particle number concentrations active as CCN at $0.01 \%, 0.1 \%, 2.8 \%$, and $5.3 \% S S_{w}$. 


\section{Appendix C: Clustering Analysis}

Clustering was done after evaluating several different methods and options. The primary parameters that were varied were the number of clusters used, and the distance metric used to distinguish clusters. All analysis was performed using built-in Matlab functions. Three distance metrics were evaluated using the "kmeans" function options: 1) squared Euclidian, 2) sum of absolute differences (cityblock), and 3) cosine. Each defines cluster distance, d, as follows:

$$
\begin{aligned}
& d_{1}(x, c)=(x-c)(x-c)^{\prime} \\
& d_{2}(x, c)=\sum_{j}\left|x_{j}-c_{j}\right|
\end{aligned}
$$

455 where $\mathrm{x}$ is input data, and $\mathrm{c}$ is a cluster. Both $\mathrm{x}$ and $\mathrm{c}$ are arrays, with subscripts indicating a single array element and apostrophes indicating a transpose operation. It was found that metrics 1 and 2 produced separation of spectra based solely on total particle number, whether clustering was applied to aerosol number size distributions or to CCN spectra. Distance metric 3, however, produced well-resolved clusters based on distribution shape, and was selected for final cluster designations. Clustering in CCN space also produced well resolved clusters in size distribution space, and vice versa. CCN space was 460 ultimately chosen for clustering, due to the focus of the current work, but differences from the alternative are expected to be very minor.

Next, optimal number of clusters was explored. While this is often accomplished somewhat arbitrarily, intuitively, or based on external models for a given process, statistical methods have been developed to guide the process. We chose to use the "gap statistic" (Tibshirani et al., 2001), a built in Matlab functionality through the "evalclusters" function. This method 465 was too computationally intensive to use on the entire data set, so a subset of 500 randomly selected spectra were used instead. Three clusters were suggested to be the optimal number to use, based on this approach. These clusters all appeared physically distinct, as discussed in Section 3.1.2, and addition of a $4^{\text {th }}$ cluster simply resulted in the splitting of two adjacent clusters. Three clusters were thus chosen for use in further analysis.

Clusters are generally similar year round, but there is some seasonal dependence within a given cluster, as shown in Figure C1. 
https://doi.org/10.5194/acp-2021-1008

Preprint. Discussion started: 13 December 2021

(c) Author(s) 2021. CC BY 4.0 License.

(c) (1)

Atmospheric

Chemistry

and Physics

Discussions
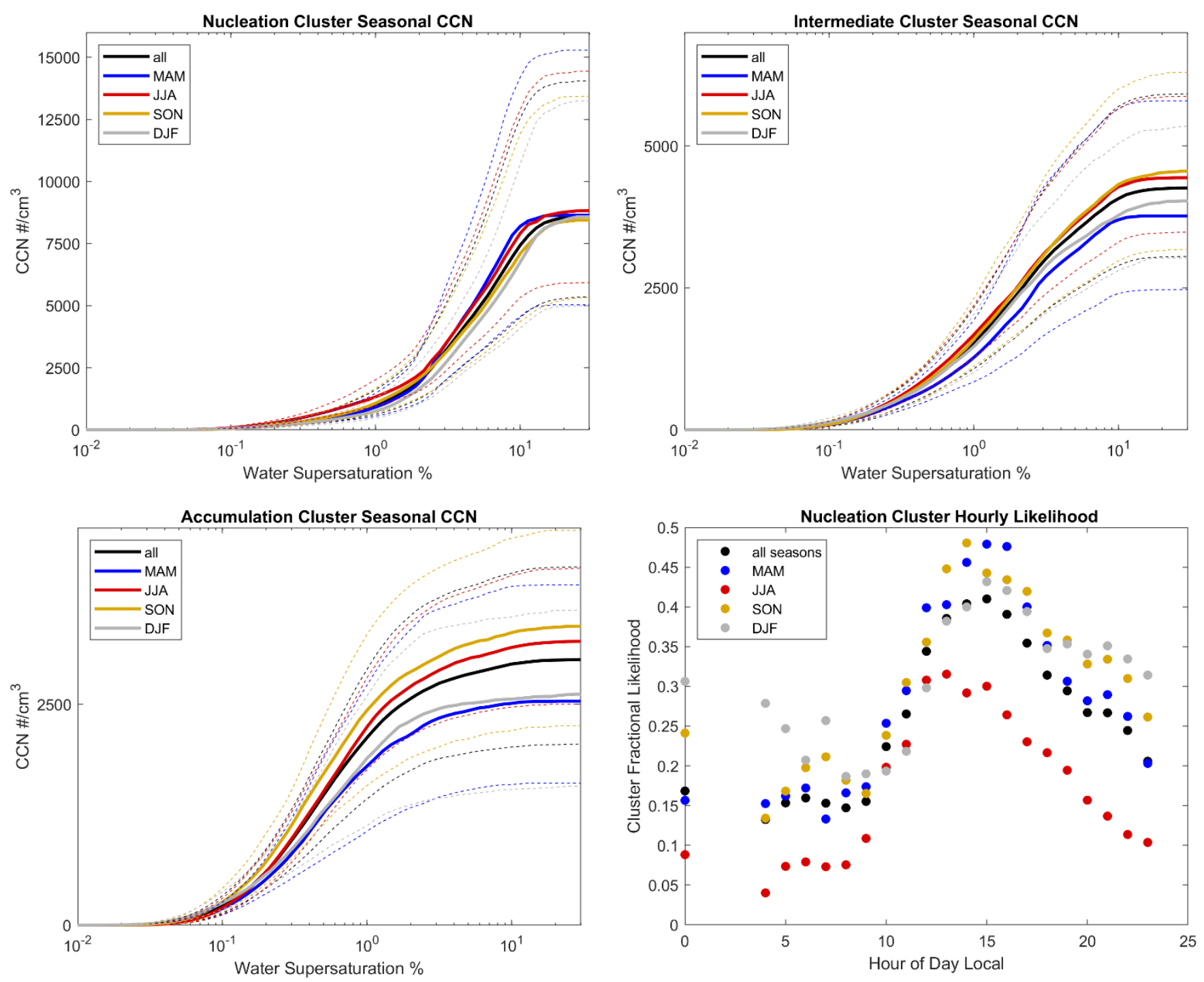

Figure C1: Median (solid lines) and confidence intervals containing $75 \%$ of the data (dotted lines) for each cluster as a function of season, along with seasonal variations in hourly cluster likelihood for the nucleation mode cluster (bottom right).

\section{Appendix D: Non-Periodic Autocorrelation and Fits}

Autocorrelation coefficients (Box et al., 2015) were generated by comparing adjacent points in a timeseries to determine the portion of variance that can be explained by the adjacent points. For a timeseries with equally spaced measurements, the autocorrelation function is defined as: 


$$
r_{k}=\frac{\sum_{i=1}^{N-k}\left(Y_{i}-\bar{Y}\right)\left(Y_{i+k}-\bar{Y}\right)}{\sum_{i=1}^{N}\left(Y_{i}-\bar{Y}\right)^{2}}
$$

Eq. 10

where $\mathrm{r}_{\mathrm{k}}$ is the autocorrelation coefficient at time lag $k, \mathrm{~N}$ is the total number of timepoints, $\mathrm{Y}_{\mathrm{i}}$ is the measurement value (in our case CCN number concentration) at timepoint $i$, and $\bar{Y}$ is the mean measurement value. For timepoints that are not evenly spaced, the same coefficient can be produced with a few extra steps. The way we have accomplished this is to 1) calculate differences between all adjacent timepoints, for a fixed number of integer time lags, before 2) sorting all of these data into time bins based on how much time elapsed between any given set of measurements.

Autocorrelation functions were subsequently fit to bi-exponential decays for the first 12 hours of time lag data, using the following form:

$$
f(x)=c_{1} e^{-\tau_{1} x}+c_{2} e^{-\tau_{2} x}
$$

Eq. 11

485 This is accomplished using the "fit" function in Matlab, which provides 95\% confidence interval information. Best fit parameters and 95\% confidence intervals are reported in Table D1 below.

Table D1: Bi-exponential Fit Coefficients

\begin{tabular}{|c|c|c|c|}
\hline & $\mathbf{0 . 1 \%} \boldsymbol{S} \boldsymbol{S}_{w}$ & $\mathbf{1 \%} \boldsymbol{S} \boldsymbol{S}_{w}$ & $\mathbf{1 0 \%} \boldsymbol{S} \boldsymbol{S}_{w}$ \\
\hline $\mathbf{c}_{\mathbf{1}}$ & $0.48(0.34,0.61)$ & $0.46(0.31,0.61)$ & $0.39(-0.09,0.88)$ \\
\hline $\boldsymbol{\tau}_{1}$ & $0.036(0.001,0.071)$ & $0.070(0.027,0.113)$ & $0.215(0.026,0.404)$ \\
\hline $\mathbf{c}_{2}$ & $0.55(0.37,0.72)$ & $0.57(0.38,0.75)$ & $0.63(0.15,1.11)$ \\
\hline $\boldsymbol{\tau}_{2}$ & $1.11(0.35,1.86)$ & $1.18(0.39,1.96)$ & $0.99(0.08,1.90)$ \\
\hline
\end{tabular}

\section{Data Availability}

490 All data are publicly available via the U.S. Department of Energy's Atmospheric Radiation Measurement (ARM) user facility data archive (Atmospheric Radiation Measurement (ARM), 1995, 2001, 2007, 2010, 2011, 2015) (https://doi.org/10.5439/1025220, https://doi.org/10.5439/1095386, https://doi.org/10.5439/1025259, https://doi.org/10.5439/1150275, https://doi.org/10.5439/1025152, and https://doi.org/10.5439/1025303), including the final merged aerosol number size distribution and CCN data (https://doi.org/10.5439/1832908). 


\section{Supplement link}

A supplemental text document containing skewed log-normal fit coefficients for all CCN data, named CCN_fit_coeffs.txt, is available at XXX.

\section{Author Contribution}

RJP performed the analyses presented. All authors assisted in interpretation of raw instrument data and construction of the merged CCN product. RJP and SMK prepared the manuscript with contributions from all co-authors.

\section{Competing Interests}

The authors declare that they have no conflicts of interest.

\section{Acknowledgments}

All data were obtained from the Atmospheric Radiation Measurement (ARM) Program sponsored by the U.S. Department of 505 Energy, Office of Science, Office of Biological and Environmental Research, Climate and Environmental Sciences Division. We would also like to acknowledge Jeffrey Pierce for helpful discussions about new particle formation events.

\section{Financial Support}

This research has been supported by the U.S. Department of Energy's Atmospheric System Research, and Office of Science, Office of Biological and Environmental Research program (grant no. DESC0016051).

\section{References}

Alexandrov, M. D., Marshak, A., Cairns, B., Lacis, A. A., and Carlson, B. E.: Scaling Properties of Aerosol Optical Thickness Retrieved from Ground-Based Measurements, J. Atmospheric Sci., 61, 1024-1039, https://doi.org/10.1175/15200469(2004)061<1024:SPOAOT>2.0.CO;2, 2004.

Alexandrov, M. D., Geogdzhayev, I. V., Tsigaridis, K., Marshak, A., Levy, R., and Cairns, B.: New Statistical Model for 515 Variability of Aerosol Optical Thickness: Theory and Application to MODIS Data over Ocean, J. Atmospheric Sci., 73, 821837, https://doi.org/10.1175/JAS-D-15-0130.1, 2016.

Anderson, T. L., Charlson, R. J., Winker, D. M., Ogren, J. A., and Holmén, K.: Mesoscale Variations of Tropospheric Aerosols, J. Atmospheric Sci., 60, 119-136, https://doi.org/10.1175/1520-0469(2003)060<0119:MVOTA>2.0.CO;2, 2003. 
Bianchi, F., Tröstl, J., Junninen, H., Frege, C., Henne, S., Hoyle, C. R., Molteni, U., Herrmann, E., Adamov, A., Bukowiecki, N., Chen, X., Duplissy, J., Gysel, M., Hutterli, M., Kangasluoma, J., Kontkanen, J., Kürten, A., Manninen, H. E., Münch, S., Peräkylä, O., Petäjä, T., Rondo, L., Williamson, C., Weingartner, E., Curtius, J., Worsnop, D. R., Kulmala, M., Dommen, J., and Baltensperger, U.: New particle formation in the free troposphere: A question of chemistry and timing, Science, 2016.

Box, G. E. P., Jenkins, G. M., Reinsel, G. C., and Ljung, G. M.: Time Series Analysis: Forecasting and Control, 5th Edition., John Wiley and Sons Inc., Hoboken, New Jersey, 712 pp., 2015.

525 Carrico, C. M., Petters, M. D., Kreidenweis, S. M., Sullivan, A. P., McMeeking, G. R., Levin, E. J. T., Engling, G., Malm, W. C., and Collett, J. L. J.: Water uptake and chemical composition of fresh aerosols generated in open burning of biomass, Atmospheric Chem. Phys., 10, 5165-5178, https://doi.org/10.5194/acp-10-5165-2010, 2010.

Cheng, W. Y. Y., Carrió, G. G., Cotton, W. R., and Saleeby, S. M.: Influence of cloud condensation and giant cloud condensation nuclei on the development of precipitating trade wind cumuli in a large eddy simulation, J. Geophys. Res. Atmospheres, 114, https://doi.org/10.1029/2008JD011011, 2009.

Cohard, J.-M., Pinty, J.-P., and Bedos, C.: Extending Twomey's Analytical Estimate of Nucleated Cloud Droplet Concentrations from CCN Spectra, J. Atmospheric Sci., 55, 3348-3357, https://doi.org/10.1175/15200469(1998)055<3348:ETSAEO>2.0.CO;2, 1998.

Collins, D.: Tandem Differential Mobility Analyzer/Aerodynamic Particle Sizer (APS) Handbook, PNNL; Richland, WA, https://doi.org/10.2172/982072, 2010.

DeCarlo, P. F., Slowik, J. G., Worsnop, D. R., Davidovits, P., and Jimenez, J. L.: Particle Morphology and Density Characterization by Combined Mobility and Aerodynamic Diameter Measurements. Part 1: Theory, Aerosol Sci. Technol., 38, 1185-1205, https://doi.org/10.1080/027868290903907, 2004.

Feingold, G., Cotton, W. R., Kreidenweis, S. M., and Davis, J. T.: The Impact of Giant Cloud Condensation Nuclei on Drizzle 540 Formation in Stratocumulus: Implications for Cloud Radiative Properties, J. Atmospheric Sci., 56, 4100-4117, https://doi.org/10.1175/1520-0469(1999)056<4100:TIOGCC>2.0.CO;2, 1999.

Gantt, B., He, J., Zhang, X., Zhang, Y., and Nenes, A.: Incorporation of advanced aerosol activation treatments into CESM/CAM5: model evaluation and impacts on aerosol indirect effects, Atmospheric Chem. Phys., 14, 7485-7497, https://doi.org/10.5194/acp-14-7485-2014, 2014.

545 Gerber, H.: Supersaturation and Droplet Spectral Evolution in Fog, J. Atmospheric Sci., 48, 2569-2588, https://doi.org/10.1175/1520-0469(1991)048<2569:SADSEI>2.0.CO;2, 1991.

Glenn, I. B., Feingold, G., Gristey, J. J., and Yamaguchi, T.: Quantification of the Radiative Effect of Aerosol-Cloud Interactions in Shallow Continental Cumulus Clouds, J. Atmospheric Sci., 77, 2905-2920, https://doi.org/10.1175/JAS-D-190269.1, 2020.

550 Hodshire, A. L., Lawler, M. J., Zhao, J., Ortega, J., Jen, C., Yli-Juuti, T., Brewer, J. F., Kodros, J. K., Barsanti, K. C., Hanson, D. R., McMurry, P. H., Smith, J. N., and Pierce, J. R.: Multiple new-particle growth pathways observed at the US DOE Southern Great Plains field site, Atmospheric Chem. Phys., 16, 9321-9348, https://doi.org/10.5194/acp-16-9321-2016, 2016.

Hudson, J. G., Jha, V., and Noble, S.: Drizzle correlations with giant nuclei, Geophys. Res. Lett., 38, https://doi.org/10.1029/2010GL046207, 2011. 

https://doi.org/10.1175/1520-0469(1982)039<0448:TROGAU>2.0.CO;2, 1982.

Jung, E., Albrecht, B. A., Jonsson, H. H., Chen, Y.-C., Seinfeld, J. H., Sorooshian, A., Metcalf, A. R., Song, S., Fang, M., and Russell, L. M.: Precipitation effects of giant cloud condensation nuclei artificially introduced into stratocumulus clouds, Atmospheric Chem. Phys., 15, 5645-5658, https://doi.org/10.5194/acp-15-5645-2015, 2015.

560 Levin, Z. and Cotton, W. R. (Eds.): Aerosol Pollution Impact on Precipitation: A Scientific Review, Springer Netherlands, https://doi.org/10.1007/978-1-4020-8690-8, 2009.

Low, R. D. H.: Microphysical and meteorological measurements of fog supersaturation, Tellus, 27, 507-513, https://doi.org/10.3402/tellusa.v27i5.10177, 1975.

Mahish, M. and Collins, D.: Analysis of a Multi-Year Record of Size-Resolved Hygroscopicity Measurements from a Rural

Marinescu, P. and Levin, E.: SGP Merged Aerosol Size Distribution (CPC+SMPS+APS), Atmospheric Radiation Measurement (ARM) Data Center, United States, https://doi.org/10.5439/1511037, 2019.

Marinescu, P. J., Heever, S. C. van den, Saleeby, S. M., Kreidenweis, S. M., and DeMott, P. J.: The Microphysical Roles of Lower-Tropospheric versus Midtropospheric Aerosol Particles in Mature-Stage MCS Precipitation, J. Atmospheric Sci., 74, 3657-3678, https://doi.org/10.1175/JAS-D-16-0361.1, 2017.

Marinescu, P. J., Levin, E. J. T., Collins, D., Kreidenweis, S. M., and Heever, S. C. van den: Quantifying aerosol size distributions and their temporal variability in the Southern Great Plains, USA, Atmospheric Chem. Phys., 19, 11985-12006, https://doi.org/10.5194/acp-19-11985-2019, 2019.

Nieminen, T., Kerminen, V.-M., Petäjä, T., Aalto, P. P., Arshinov, M., Asmi, E., Baltensperger, U., Beddows, D. C. S., Beukes, 575 J. P., Collins, D., Ding, A., Harrison, R. M., Henzing, B., Hooda, R., Hu, M., Hõrrak, U., Kivekäs, N., Komsaare, K., Krejci, R., Kristensson, A., Laakso, L., Laaksonen, A., Leaitch, W. R., Lihavainen, H., Mihalopoulos, N., Németh, Z., Nie, W., O’Dowd, C., Salma, I., Sellegri, K., Svenningsson, B., Swietlicki, E., Tunved, P., Ulevicius, V., Vakkari, V., Vana, M., Wiedensohler, A., Wu, Z., Virtanen, A., and Kulmala, M.: Global analysis of continental boundary layer new particle formation based on long-term measurements, Atmospheric Chem. Phys., 18, 14737-14756, https://doi.org/10.5194/acp-18-14737-2018, 2018.

Patel, P. N. and Jiang, J. H.: Cloud condensation nuclei characteristics at the Southern Great Plains site: role of particle size distribution and aerosol hygroscopicity, Environ. Res. Commun., 3, 075002, https://doi.org/10.1088/2515-7620/ac0e0b, 2021.

Petters, M. D. and Kreidenweis, S. M.: A single parameter representation of hygroscopic growth and cloud condensation nucleus activity, Atmospheric Chem. Phys., 7, 1961-1971, https://doi.org/10.5194/acp-7-1961-2007, 2007.

585 Pierce, J. R., Westervelt, D. M., Atwood, S. A., Barnes, E. A., and Leaitch, W. R.: New-particle formation, growth and climaterelevant particle production in Egbert, Canada: analysis from 1 year of size-distribution observations, Atmospheric Chem. Phys., 14, 8647-8663, https://doi.org/10.5194/acp-14-8647-2014, 2014.

Pinsky, M., Khain, A., Mazin, I., and Korolev, A.: Analytical estimation of droplet concentration at cloud base, J. Geophys. Res. Atmospheres, 117, https://doi.org/10.1029/2012JD017753, 2012. 
590 Posselt, R. and Lohmann, U.: Influence of Giant CCN on warm rain processes in the ECHAM5 GCM, Atmospheric Chem. Phys., 8, 3769-3788, https://doi.org/10.5194/acp-8-3769-2008, 2008.

Saleeby, S. M., van den Heever, S. C., Marinescu, P. J., Kreidenweis, S. M., and DeMott, P. J.: Aerosol effects on the anvil characteristics of mesoscale convective systems, J. Geophys. Res. Atmospheres, 121, 10,880-10,901, https://doi.org/10.1002/2016JD025082, 2016.

595 Sayer, A. M. and Knobelspiesse, K. D.: How should we aggregate data? Methods accounting for the numerical distributions, with an assessment of aerosol optical depth, Atmospheric Chem. Phys., 19, 15023-15048, https://doi.org/10.5194/acp-1915023-2019, 2019.

Shen, C., Zhao, C., Ma, N., Tao, J., Zhao, G., Yu, Y., and Kuang, Y.: Method to Estimate Water Vapor Supersaturation in the Ambient Activation Process Using Aerosol and Droplet Measurement Data, J. Geophys. Res. Atmospheres, 123, 10,606600 10,619, https://doi.org/10.1029/2018JD028315, 2018.

Tibshirani, R., Walther, G., and Hastie, T.: Estimating the number of clusters in a data set via the gap statistic, J. R. Stat. Soc. Ser. B Stat. Methodol., 63, 411-423, https://doi.org/10.1111/1467-9868.00293, 2001.

Uin, J.: Cloud Condensation Nuclei Particle Counter Instrument Handbook, DOE ARM Clim. Res. Facil., https://doi.org/DOE/SC-ARM-TR-168, 2016.

605 Venzac, H., Sellegri, K., Laj, P., Villani, P., Bonasoni, P., Marinoni, A., Cristofanelli, P., Calzolari, F., Fuzzi, S., Decesari, S., Facchini, M.-C., Vuillermoz, E., and Verza, G. P.: High frequency new particle formation in the Himalayas, Proc. Natl. Acad. Sci., 105, 15666-15671, https://doi.org/10.1073/pnas.0801355105, 2008. 